\title{
Entropy of Quantum Black Holes ${ }^{\star}$
}

Romesh K. KAUL

The Institute of Mathematical Sciences, CIT Campus, Chennai-600 113, India

E-mail: kaul@imsc.res.in

Received September 14, 2011, in final form February 03, 2012; Published online February 08, 2012

http://dx.doi.org/10.3842/SIGMA.2012.005

\begin{abstract}
In the Loop Quantum Gravity, black holes (or even more general Isolated Horizons) are described by a $S U(2)$ Chern-Simons theory. There is an equivalent formulation of the horizon degrees of freedom in terms of a $U(1)$ gauge theory which is just a gauged fixed version of the $S U(2)$ theory. These developments will be surveyed here. Quantum theory based on either formulation can be used to count the horizon micro-states associated with quantum geometry fluctuations and from this the micro-canonical entropy can be obtained. We shall review the computation in $S U(2)$ formulation. Leading term in the entropy is proportional to horizon area with a coefficient depending on the Barbero-Immirzi parameter which is fixed by matching this result with the Bekenstein-Hawking formula. Remarkably there are corrections beyond the area term, the leading one is logarithm of the horizon area with a definite coefficient $-3 / 2$, a result which is more than a decade old now. How the same results are obtained in the equivalent $U(1)$ framework will also be indicated. Over years, this entropy formula has also been arrived at from a variety of other perspectives. In particular, entropy of BTZ black holes in three dimensional gravity exhibits the same logarithmic correction. Even in the String Theory, many black hole models are known to possess such properties. This suggests a possible universal nature of this logarithmic correction.
\end{abstract}

Key words: black holes; micro-canonical entropy; topological field theories; $S U(2)$ ChernSimons theory; Isolated Horizons; Bekenstein-Hawking formula; logarithmic correction; Barbero-Immirzi parameter; conformal field theories; Cardy formula; BTZ black hole; canonical entropy

2010 Mathematics Subject Classification: 81T13; 81T45; 83C57; 83C45; 83C47

\section{Introduction}

Black holes have fascinated the imagination of physicists and astronomers for a long time now. There is mounting astronomical evidence for objects with black hole like properties; in fact, these may occur abundantly in the Universe. Theoretical studies of black hole properties have been pursued, both at the classical level and traditionally at semi-classical level, for a long time. The pioneering work of Bekenstein, Hawking and others during seventies of the last century have suggested that black holes are endowed with thermodynamic attributes such as entropy and temperature [1]. Semi-classical arguments have led to the fact that this entropy is very large and is given, in the natural units, by a quarter of the horizon area, the Bekenstein-Hawking area law. Understanding these properties is a fundamental challenge within the framework of a full fledged theory of quantum gravity. The entropy would have its origin in the quantum gravitational micro-states associated with the horizon. In fact reproducing these thermodynamic properties of black holes can be considered as a possible test of such a quantum theory.

There are several proposals for theory of quantum gravity. Two of these are the String Theory and the Loop Quantum Gravity. There are other theories like dynamical triangulations and also Sorkins's causal set framework. Here we shall survey some of the developments regarding

\footnotetext{
*This paper is a contribution to the Special Issue "Loop Quantum Gravity and Cosmology". The full collection is available at http://www.emis.de/journals/SIGMA/LQGC.html
} 
black hole entropy within a particular theory of quantum gravity, the Loop Quantum Gravity (LQG) where the degrees of freedom of the event horizon of a black hole are described by a quantum $S U(2)$ Chern-Simons theory. This also holds for the more general horizons, the Isolated Horizons of Ashtekar et al. [2], which, defined quasi-locally, have been introduced to describe situations like a black hole in equilibrium with its dynamical exterior. Not only is the semi-classical Bekenstein-Hawking area law reproduced for a large hole, quantum microcanonical entropy has additional corrections which depend on the logarithm of horizon area with a definite, possibly universal, coefficient $-3 / 2$, followed by an area independent constant and terms which are inverse powers of area. Presence of these additional corrections is the hallmark of quantum geometry. These results, first derived within LQG framework in four dimensions, have also been seen to emerge in other contexts. For example, the entropy of BTZ black holes in three-dimensional gravity displays similar properties. Additionally, application of the Cardy formula of conformal field theories, which are relevant to study black holes in the String Theory, also implies such corrections to the area law. Though the main thrust of this article is to survey developments in LQG, we shall also review, though only briefly, a few calculations of black hole entropy from other perspectives.

\section{Horizon topological field theory}

That the horizon degrees of freedom of a black hole are described by a $S U(2)$ topological field theory follows readily from following two facts [3]:

(i) The event horizon (EH) of a black hole space-time (and more generally an Isolated Horizon (IH) [2]), is a null inner boundary of the space-time accessible to an asymptotic observer. It has the topology $\mathbb{R} \times \mathbb{S}^{2}$ and a degenerate intrinsic three-metric. Consequently, such a manifold can not support any local propagating degree of freedom which would, otherwise, have to be described by a Lagrangian density containing determinant and inverse of the metric. The horizon degrees of freedom have to be entirely global or topological. These can be described only by a theory which does not depend on the metric, a topological quantum field theory ${ }^{1}$.

(ii) In the Loop Quantum Gravity framework, bulk space-time properties are described in terms of Sen-Ashtekar-Barbero-Immirzi real SU(2) connections [6]. Physics associated with bulk space-time geometry is invariant under local $S U(2)$ transformations. The EH (more generally the $\mathrm{IH}$ ) is a null boundary where Einstein's equation holds. At the classical level, the degrees of freedom and their dynamics on an EH (IH) are completely determined by the geometry and dynamics in the bulk. Quantum theory of horizon degrees of freedom has to imbibe this $S U(2)$ gauge invariance from the bulk.

In view of these two properties, degrees of freedom associated with a horizon have to be described by a topological field theory exhibiting $S U(2)$ gauge invariance. There are two such three-dimensional candidates, the Chern-Simons and BF theories. However, both these theories essentially capture the same topological properties [5] and hence would provide equivalent descriptions. It is, therefore, no surprise that when the detail properties of the various geometric quantities on the horizon are analysed, as has been done in several places in literature, they are found to obey equations of motion of the topological $S U(2)$ Chern-Simons theory (or equivalently BF theory) with specific sources on the three-manifold $\mathbb{R} \times \mathbb{S}^{2}$. This description can be presented either in the form of a theory with full fledged $S U(2)$ gauge invariance or, equivalently, by a gauge fixed $U(1)$ theory. We shall review this in Section 2.1 below for the Schwarzschild hole. Similar results hold for the more general case of Isolated Horizons [2], which shall be briefly summarized next in the Section 2.2. The sources of the Chern-Simons theory are constructed from tetrad components in the bulk. Clearly, quantizing this Chern-Simons

\footnotetext{
${ }^{1}$ For reviews of topological field theories see, for example $[4,5]$.
} 
theory paves a way for counting micro-states of the horizon and hence the associated entropy which we shall take up in Section 3.

\subsection{Schwarzschild black hole}

Following closely the analysis of [3], we shall study the properties of future event horizon of the Kruskal-Szekeres extension of Schwarzschild space-time explicitly. In the process, we shall unravel the relationship between the horizon $S U(2)$ and $U(1)$ Chern-Simons theories. We display an appropriate set of tetrad fields, which finally leads to the gauge fields on the black hole (future) horizon with only manifest $U(1)$ invariance. For this choice, we find that two components of $S U(2)$ triplet solder forms on the spatial slice of the horizon, orthogonal to the direction specified by the $U(1)$ subgroup, are indeed zero as they should be. This is in agreement with the general analysis of [7]. In the next subsection, we explicitly demonstrate how the equations of motion of $U(1)$ theory so obtained are to be interpreted as those coming from a $S U(2)$ Chern-Simons theory through a partial gauge fixing procedure. In the course of our analysis, we also derive the dependence of coupling constant of these Chern-Simons theories on the Barbero-Immirzi parameter $\gamma$ and horizon area $A_{\mathrm{H}}$.

Schwarzschild metric in the Kruskal-Szekeres null coordinates $v$ and $w$ is given by its nonzero components as: $g_{v w}=g_{w v}=-\left(4 r_{0}^{3} / r\right) \exp \left(-r / r_{0}\right), g_{\theta \theta}=r^{2}, g_{\phi \phi}=r^{2} \sin \theta$. Here $r$ is a function of $v$ and $w$ through: $-2 v w=\left[\left(r / r_{0}\right)-1\right] \exp \left(r / r_{0}\right)$. An appropriate set of tetrad fields compatible with this metric in the exterior region of the black hole $(v>0, w<0)$ is:

$$
\begin{aligned}
e_{\mu}^{0} & =\sqrt{\frac{A}{2}}\left(\frac{w}{\alpha} \partial_{\mu} v+\frac{\alpha}{w} \partial_{\mu} w\right), & e_{\mu}^{1} & =\sqrt{\frac{A}{2}}\left(\frac{w}{\alpha} \partial_{\mu} v-\frac{\alpha}{w} \partial_{\mu} w\right), \\
e_{\mu}^{2} & =r \partial_{\mu} \theta, & e_{\mu}^{3} & =r \sin \theta \partial_{\mu} \phi,
\end{aligned}
$$

where $A \equiv\left(4 r_{0}^{3} / r\right) \exp \left(-r / r_{0}\right)$ and $\alpha$ is an arbitrary function of the coordinates. A choice of $\alpha(x)$ characterizes the local Lorentz frame in the indefinite metric plane $\mathcal{I}$ of the Schwarzschild spacetime whose spherical symmetry implies that it has the topology $\mathcal{I} \otimes \mathbb{S}^{2}$. The spin connections can be constructed for this set of tetrads to be:

$$
\begin{aligned}
& \omega_{\mu}^{01}=-\frac{1}{2}\left(1-\frac{r_{0}^{2}}{r^{2}}\right) \frac{1}{v} \partial_{\mu} v-\frac{1}{2}\left(1+\frac{r_{0}^{2}}{r^{2}}\right) \frac{1}{w} \partial_{\mu} w+\partial_{\mu} \ln \alpha, \quad \omega_{\mu}^{23}=-\cos \theta \partial_{\mu} \phi, \\
& \omega_{\mu}^{02}=-\sqrt{\frac{A}{2}} \frac{1}{2 r_{0}}\left(\frac{v w}{\alpha}+\alpha\right) \partial_{\mu} \theta, \quad \omega_{\mu}^{03}=-\sqrt{\frac{A}{2}} \frac{\sin \theta}{2 r_{0}}\left(\frac{v w}{\alpha}+\alpha\right) \partial_{\mu} \phi, \\
& \omega_{\mu}^{12}=-\sqrt{\frac{A}{2}} \frac{1}{2 r_{0}}\left(\frac{v w}{\alpha}-\alpha\right) \partial_{\mu} \theta, \quad \omega_{\mu}^{13}=-\sqrt{\frac{A}{2}} \frac{\sin \theta}{2 r_{0}}\left(\frac{v w}{\alpha}-\alpha\right) \partial_{\mu} \phi .
\end{aligned}
$$

LQG is described in terms of linear combinations of these connection components involving the Barbero-Immirzi parameter $\gamma$, the real Sen-Ashtekar-Barbero-Immirzi $S U(2)$ gauge fields [6]. To this effect, we construct the $S U(2)$ gauge fields:

$$
A_{\mu}^{(i)}=\gamma \omega_{\mu}^{0 i}-\frac{1}{2} \epsilon^{i j k} \omega_{\mu}^{j k} .
$$

The black hole horizon is the future horizon given by $w=0$. This is a null three-manifold $\Delta$ which is topologically $\mathbb{R} \times \mathbb{S}^{2}$ and is described by the coordinates $a=(v, \theta, \phi)$ with $0<v<\infty$, $0 \leq \theta<\pi, 0 \leq \phi<2 \pi$. The foliation of manifold $\Delta$ is provided by $v=$ constant surfaces, each an $\mathbb{S}^{2}$. The relevant tetrad fields $e_{a}^{I}$ on the horizon $\Delta$ from (2.1) are: $e_{a}^{0} \hat{=} 0, e_{a}^{1} \hat{=} 0$, $e_{a}^{2} \hat{=} r_{0} \partial_{a} \theta, e_{a}^{3} \hat{=} r_{0} \sin \theta \partial_{a} \phi$ where $a=(v, \theta, \phi)$ (we denote equalities on $\Delta$, that is for $w=0$, by the symbol $\hat{=})$. The intrinsic metric on $\Delta$ is degenerate with its signature $(0,+,+)$ and is given by $q_{a b}=e_{a}^{I} e_{I b} \hat{=} m_{a} \bar{m}_{b}+m_{b} \bar{m}_{a}$ where $m_{a} \equiv r_{0}\left(\partial_{a} \theta+i \sin \theta \partial_{a} \phi\right) / \sqrt{2}$. 
Only non-zero solder form $\Sigma_{a b}^{I J} \equiv e_{[a}^{I} e_{b]}^{J}$ on the horizon is $\Sigma_{a b}^{23} \hat{=} r_{0}^{2} \sin \theta \partial_{[a} \theta \partial_{b]} \phi$. The spin connection fields from (2.2) are:

$$
\begin{array}{lll}
\omega_{a}^{01} \hat{=} \frac{1}{2} \partial_{a} \ln \beta, & \omega_{a}^{02} \hat{=}-\sqrt{\beta} \partial_{a} \theta, & \omega_{a}^{03} \hat{=}-\sqrt{\beta} \sin \theta \partial_{a} \phi \\
\omega_{a}^{23} \hat{=}-\cos \theta \partial_{a} \phi, & \omega_{a}^{31} \hat{=}-\sqrt{\beta} \sin \theta \partial_{a} \phi, & \omega_{a}^{12} \hat{=} \sqrt{\beta} \partial_{a} \theta
\end{array}
$$

where $\beta=\alpha^{2} /(2 e)$ with $e \equiv \exp (1)$. Notice that the spin connection field $\omega_{a}^{01}=\frac{1}{2} \partial_{a} \ln \beta$ here, with a possible singular behaviour for $\beta=0$, is a pure gauge. If we wish, by a suitable boost transformation $\omega_{a}^{I J} \rightarrow \omega_{a}^{\prime I J}$, it can be rotated away to zero, with corresponding changes in other spin connection fields: $\omega_{a}^{\prime 01}=\omega_{a}^{01}-\partial_{a} \xi, \omega_{a}^{\prime 23}=\omega_{a}^{23}, \omega_{a}^{\prime 02}=\cosh \xi \omega_{a}^{02}+\sinh \xi \omega_{a}^{12}, \omega_{a}^{\prime 03}=$ $\cosh \xi \omega_{a}^{03}+\sinh \xi \omega_{a}^{13}, \omega_{a}^{\prime 12}=\sinh \xi \omega_{a}^{02}+\cosh \xi \omega_{a}^{12}$, and $\omega_{a}^{\prime 13}=\sinh \xi \omega_{a}^{03}+\cosh \xi \omega_{a}^{13}$. For the choice $\xi=\frac{1}{2} \ln \left(\beta / \beta^{\prime}\right)$ with $\beta^{\prime}$ as an arbitrary constant, this leads to $\omega_{a}^{\prime 01} \hat{=} 0, \omega_{a}^{\prime 23} \hat{=}-\cos \theta \partial_{a} \phi$, $\omega_{a}^{\prime 02} \hat{=}-\sqrt{\beta^{\prime}} \partial_{a} \theta, \omega_{a}^{\prime 03} \hat{=}-\sqrt{\beta^{\prime}} \sin \theta \partial_{a} \phi, \omega_{a}^{\prime 12} \hat{=} \sqrt{\beta^{\prime}} \partial_{a} \theta$ and $\omega_{a}^{\prime 13} \hat{=} \sqrt{\beta^{\prime}} \sin \theta \partial_{a} \phi$.

To demonstrate that the horizon degrees of freedom can be described by a Chern-Simons theory, we use (2.4) to write the relevant components of $S U(2)$ gauge fields (2.3) on $\Delta$ as:

$$
\begin{aligned}
& A_{a}^{(1)} \hat{=} \frac{\gamma}{2} \partial_{a} \ln \beta+\cos \theta \partial_{a} \phi, \quad A_{a}^{(2)} \hat{=}-\sqrt{\beta}\left(\gamma \partial_{a} \theta-\sin \theta \partial_{a} \phi\right), \\
& A_{a}^{(3)} \hat{=}-\sqrt{\beta}\left(\gamma \sin \theta \partial_{a} \phi+\partial_{a} \theta\right) .
\end{aligned}
$$

The field strength components constructed from these satisfy the following relations on $\Delta$ :

$$
\begin{aligned}
& F_{a b}^{(1)} \equiv 2 \partial_{[a} A_{b]}^{(1)}+2 A_{[a}^{(2)} A_{b]}^{(3)} \hat{=}-\frac{2}{r_{0}^{2}}\left(1-K^{2}\right) \Sigma_{a b}^{23}, \\
& F_{a b}^{(2)} \equiv 2 \partial_{[a} A_{b]}^{(2)}+2 A_{[a}^{(3)} A_{b]}^{(1)} \hat{=}-2 \sqrt{1+\gamma^{2}} \sin \theta \partial_{[a} \phi \partial_{b]} K, \\
& F_{a b}^{(3)} \equiv 2 \partial_{[a} A_{b]}^{(3)}+2 A_{[a}^{(1)} A_{b]}^{(2)} \hat{=} 2 \sqrt{1+\gamma^{2}} \partial_{[a} \theta \partial_{b]} K,
\end{aligned}
$$

where $\Sigma_{\mu \nu}^{I J}=e_{[\mu}^{I} e_{\nu]}^{J} \equiv \frac{1}{2}\left(e_{\mu}^{I} e_{\nu}^{J}-e_{\nu}^{I} e_{\mu}^{J}\right)$ and $K=\sqrt{\beta\left(1+\gamma^{2}\right)}$ with $\beta$ as an arbitrary function of space-time coordinates. We may gauge fix the invariance under boost transformations by a convenient choice of $\beta$ as follows:

Case (i): A choice of basis is provided by $\beta \equiv \alpha^{2} /(2 e)=-v w /(2 e) \hat{=} 0(K \hat{=} 0)$. For this choice, the $S U(2)$ gauge fields from (2.5) are:

$$
A_{a}^{(1)} \hat{=} \frac{\gamma}{2} \partial_{a} \ln v+\cos \theta \partial_{a} \phi, \quad A_{a}^{(2)} \hat{=} 0, \quad A_{a}^{(3)} \hat{=} 0
$$

and equations (2.6) lead to

$$
F_{a b}^{(1)} \hat{=} 2 \partial_{[a} A_{b]}^{(1)} \hat{=}-\frac{2}{r_{0}^{2}} \Sigma_{a b}^{23}=-\frac{2 \gamma}{r_{0}^{2}} \Sigma_{a b}^{(1)}, \quad F_{a b}^{(2)} \hat{=} 0, \quad F_{a b}^{(3)} \hat{=} 0 .
$$

These relations are invariant under $U(1)$ transformations: $A_{a}^{(1)} \rightarrow A_{a}^{(1)}-\partial_{a} \xi$ with $A_{a}^{(2)}$ and $A_{a}^{(3)}$ unaltered. As we shall show in the next subsection, these relations can be interpreted as the equations of motion of a $S U(2)$ Chern-Simons theory gauge fixed to a $U(1)$ theory.

The $U(1)$ Chern-Simons action for which the first relation in (2.8) is the Euler-Lagrangian equation of motion, may be written as:

$$
S_{1}=\frac{k}{4 \pi} \int_{\Delta} \epsilon^{a b c} A_{a} \partial_{b} A_{c}+\int_{\Delta} J^{a} A_{a}
$$

where the non-zero components of the completely antisymmetric $\epsilon^{a b c}$ are given by $\epsilon^{v \theta \phi}=1$ and $A_{a} \equiv A_{a}^{(1)}$ is the $U(1)$ gauge field. The external source is given by the vector density with 
upper index $a$ as: $J^{a}=\epsilon^{a b c} \Sigma_{b c}^{(1)} / 2$. The coupling is directly proportional to the horizon area and inversely to the Barbero-Immirzi parameter: $k=\pi r_{0}^{2} / \gamma \equiv A_{\mathrm{H}} /(4 \gamma)$.

Case (ii): On the other hand, we could make another gauge choice where $\beta$ is constant ( $K=\sqrt{\beta\left(1+\gamma^{2}\right)}=$ constant) but arbitrary, with gauge fields given by:

$$
\begin{aligned}
& A_{a}^{(1)} \hat{=} \cos \theta \partial_{a} \phi, \quad A_{a}^{(2)} \hat{=}-K\left(\cos \delta \partial_{a} \theta-\sin \delta \sin \theta \partial_{a} \phi\right), \\
& A_{a}^{(3)} \hat{=}-K\left(\sin \delta \partial_{a} \theta+\cos \delta \sin \theta \partial_{a} \phi\right),
\end{aligned}
$$

where $\cot \delta=\gamma$. The field strength components (2.6) satisfy:

$$
\begin{aligned}
& F_{a b}^{(1)} \equiv 2 \partial_{[a} A_{b]}^{(1)}+2 A_{[a}^{(2)} A_{b]}^{(3)} \hat{=}-\frac{2 \gamma}{r_{0}^{2}}\left[1-\beta\left(1+\gamma^{2}\right)\right] \Sigma_{a b}^{(1)}, \\
& F_{a b}^{(2)} \equiv 2 \partial_{[a} A_{b]}^{(2)}+2 A_{[a}^{(3)} A_{b]}^{(1)} \hat{=} 0, \quad F_{a b}^{(3)} \equiv 2 \partial_{[a} A_{b]}^{(3)}+2 A_{[a}^{(1)} A_{b]}^{(2)} \hat{=} 0 .
\end{aligned}
$$

These equations have invariance under $U(1)$ transformations: $A_{a}^{(1)} \rightarrow A_{a}^{(1)}-\partial_{a} \xi, A_{a}^{(2)} \rightarrow$ $\cos \xi A_{a}^{(2)}+\sin \xi A_{a}^{(3)}$ and $A_{a}^{(3)} \rightarrow-\sin \xi A_{a}^{(2)}+\cos \xi A_{a}^{(3)}$. This reflects that the field $A_{a}^{(1)}$ is a $U(1)$ gauge field and fields $A_{a}^{(2)}$ and $A_{a}^{(3)}$ are an $O(2)$ doublet with $U(1)$ transformations acting as a rotation on them.

Identifying $U(1)$ gauge field as $A_{a} \equiv A_{a}^{(1)}$ and defining the complex vector fields $\phi_{a}=$ $\left(A_{a}^{(2)}+i A_{a}^{(3)}\right) / \sqrt{2}$ and $\bar{\phi}_{a}=\left(A_{a}^{(2)}-i A_{a}^{(3)}\right) / \sqrt{2}$, the relations $(2.11)$ can be recast as:

$$
F_{a b}-2 i \bar{\phi}_{[a} \phi_{b]} \hat{=}-\frac{2 \gamma}{r_{0}^{2}}\left[1-\beta\left(1+\gamma^{2}\right)\right] \Sigma_{a b}^{(1)}, \quad D_{[a}(A) \phi_{b]} \hat{=} 0, \quad D_{[a}(A) \bar{\phi}_{b]} \hat{=} 0
$$

where the $U(1)$ field strength is $F_{a b} \equiv 2 \partial_{[a} A_{b]}$ and covariant derivatives of the charged vector fields are $D_{a}(A) \phi_{b} \equiv\left(\partial_{a}+i A_{a}\right) \phi_{b}$ and $D_{a}(A) \bar{\phi}_{b} \equiv\left(\partial_{a}-i A_{a}\right) \bar{\phi}_{b}$ reflecting that $\phi_{a}$ possesses one unit of $U(1)$ charge. Now an action principle that would yield (2.12) as its equations of motion can be written as:

$$
S_{2}=\frac{k}{4 \pi} \int_{\Delta} \epsilon^{a b c}\left[A_{a} \partial_{b} A_{c}+\bar{\phi}_{a} D_{b}(A) \phi_{c}+\phi_{a} D_{b}(A) \bar{\phi}_{c}\right]+\int_{\Delta} J^{a} A_{a}
$$

where $k=\pi r_{0}^{2} / \gamma \equiv A_{\mathrm{H}} /(4 \gamma)$ is the coupling and $J^{a} \equiv\left[1-\beta\left(1+\gamma^{2}\right)\right] \epsilon^{a b c} \Sigma_{b c}^{(1)} / 2$ is the external source. There is an arbitrary constant gauge parameter $\beta$ in the source which can be changed by a boost transformation of the original tetrad fields. Notice that for $\beta=\left(1+\gamma^{2}\right)^{-1}$, the source vanishes. The topological field theory described by action (2.13) is invariant under $U(1)$ transformations: $A_{a} \rightarrow A_{a}-\partial_{a} \xi, \phi_{a} \rightarrow e^{i \xi} \phi_{a}$ and $\bar{\phi}_{a} \rightarrow e^{-i \xi} \bar{\phi}_{a}$.

We could interpret the equations (2.11) or the equivalent set (2.12) alternatively by taking the combination $k=\frac{A_{\mathrm{H}}}{4 \gamma\left[1-\beta\left(1+\gamma^{2}\right)\right]}$ to be the coupling and $J^{a}=\epsilon^{a b c} \Sigma_{b c}^{(1)} / 2$ as the source. This results in a gauge dependent arbitrariness in the coupling constant, reflected through the constant parameter $\beta$. Boost transformations of the original gravity fields can be used to change the value of $\beta$. In particular, for $\beta=1 / 2$, the coupling is $k=\frac{A_{\mathrm{H}}}{2 \gamma\left(1-\gamma^{2}\right)}$ and we realize the gauge theory discussed in [8].

The presence of the arbitrary parameter $\beta$ is a reflection of the ambiguity associated with gauge fixing of invariance under boost transformations of the original tetrads $e_{a}^{I}$ and connection fields $\omega_{a}^{I J}$. Like in any gauge theory, a special choice of gauge fixing only provides a convenient description of the theory. No physical quantities should depend on the ambiguity of gauge fixing. In particular, the Chern-Simons coupling constant is a physical object. As we shall see later, physical quantities such as the quantum horizon entropy depend on this coupling. This suggests that the coupling $k$ of horizon Chern-Simons theory can not depend on $\beta$ or any 
particular value for it. A formulation of the theory that exhibits such a dependence is suspect. This is to be contrasted with the dependence on the Barbero-Immirzi parameter $\gamma$ which is perfectly possible, because $\gamma$ is not a gauge parameter but a genuine coupling constant (in fact with a topological origin) of quantum gravity. This perspective, therefore, picks up the first interpretation above for the equations (2.11) or (2.12) as represented by the action (2.13) with coupling $k=\pi r_{0}^{2} / \gamma \equiv A_{\mathrm{H}} /(4 \gamma)$ as the correct one.

Notice that the factor $\left(1+\gamma^{2}\right)$ in equations (2.11) and (2.12) arises because of the presence of $\gamma$ in the gauge field combinations defined in equations (2.5). This factor does not have any special significance as it can be absorbed in the definition of the arbitrary constant boost gauge parameter $\beta$ obtaining a new boost parameter $\beta^{\prime}=\left(1+\gamma^{2}\right) \beta$. Also in equations (2.5), we could as well replace $\gamma$ by another arbitrary constant $\lambda$, finally leading to the equation (2.11) with the factor $\left(1+\gamma^{2}\right)$ replaced by $\left(1+\lambda^{2}\right)$ which again can be absorbed in the arbitrary boost gauge parameter $\beta$ without changing any of the subsequent discussion. This is to be contrasted with the overall factor of $\gamma$ in the right-hand sides of equations (2.11) and (2.12), which is not to be absorbed away in to the boost parameter, and instead becomes part of the coupling $k$ of the Chern-Simons theory in (2.13).

The boundary topological theory describing the horizon quantum degrees of freedom and the bulk quantum theory can be thought of as decoupled from each other except for the sources of the boundary theory which depend on the bulk quantum fields $\Sigma_{a b}^{(1)}$. In fact in the bulk theory, $\epsilon^{a b c} \Sigma_{b c}^{(i)} / 2$ are the canonical conjugate momentum fields for the Sen-Ashtekar-Barbero-Immirzi $S U(2)$ gauge fields $A_{a}^{(i)} \equiv \gamma \omega_{a}^{0 i}-\frac{1}{2} \epsilon^{i j k} \omega_{a}^{j k}$. On the other hand, in the boundary theory described in terms of $U(1)$ Chern-Simons theory, the fields $\left(A_{\theta}, A_{\phi}\right)$ form a canonical pair. This allows for the fact that in the classical theory, the boost gauge fixing of the original gravity fields $\left(e_{a}^{I}, \omega_{a}^{I J}\right)$ to obtain the Chern-Simons boundary theory and that in the bulk theory can be done independently. In particular, we could choose $\beta \equiv \alpha^{2} /(2 e)=-v w /(2 e) \hat{=} 0$ (or the other choice $\beta=$ const) for the boundary theory, and make another independent convenient choice for the bulk theory, in particular, say the standard time gauge, so that the resultant canonical theory in terms of Sen-Ashtekar-Barbero-Immirzi gauge fields in the bulk can, at quantum level, lead to the standard Loop Quantum Gravity theory.

After these general remarks, let us now turn to discuss how the $U(1)$ invariant equations $(2.8)$ or (2.12) can be arrived at from a general $S U(2)$ Chern-Simons theory through a gauge fixing procedure. This we do in the next subsection.

We notice that the source for resultant $U(1)$ gauge theory in either of the cases (i) and (ii) above is given in terms of $\Sigma_{a b}^{(1)} \equiv \gamma^{-1} \Sigma_{a b}^{23}$ which is one of the components of the $S U(2)$ triplet of solder forms. An important property to note here is that for both these cases, other two components of this triplet are zero on the horizon:

$$
\Sigma_{\theta \phi}^{(2)} \equiv \gamma^{-1} \Sigma_{\theta \phi}^{31} \hat{=} 0 \quad \text { and } \quad \Sigma_{\theta \phi}^{(3)} \equiv \gamma^{-1} \Sigma_{\theta \phi}^{12} \hat{=} 0,
$$

because $e_{\theta}^{1} \hat{=} 0$ and $e_{\phi}^{1} \hat{=} 0$.

\subsection{Horizon $S U(2)$ Chern-Simons theory}

The $U(1)$ gauge theories described by the two sets of equations (2.8) and (2.11) of the respective cases (i) and (ii) along with the conditions (2.14) on the solder forms, are related to a $S U(2)$ Chern-Simons theory through a partial gauge fixing [3]. To exhibit this explicitly, consider the Chern-Simons action with coupling $k$ :

$$
S_{\mathrm{CS}}=\frac{k}{4 \pi} \int_{\Delta} \epsilon^{a b c}\left(A_{a}^{\prime(i)} \partial_{b} A_{c}^{\prime(i)}+\frac{1}{3} \epsilon^{i j k} A_{a}^{\prime(i)} A_{b}^{\prime(j)} A_{c}^{\prime(k)}\right)+\int_{\Delta} J^{\prime(i) a} A_{a}^{\prime(i)}
$$


where $A_{a}^{\prime(i)}$ are the $S U(2)$ gauge fields. This is a topological field theory: the action is independent of the metric of three-manifold $\Delta$. We take the covariantly conserved $S U(2)$ triplet of sources, which are vector densities with upper index $a$, to have a special form as: $J^{\prime(i) a} \equiv\left(J^{\prime(i) v}, J^{\prime(i) \theta}, J^{\prime(i) \phi}\right)=\left(J^{\prime(i)}, 0,0\right)$.

The action (2.15) leads to the Euler-Lagrange equations of motion:

$$
F_{v \theta}^{(i)}\left(A^{\prime}\right) \hat{=} 0, \quad F_{v \phi}^{(i)}\left(A^{\prime}\right) \hat{=} 0, \quad \frac{k}{2 \pi} F_{\theta \phi}^{(i)}\left(A^{\prime}\right) \hat{=}-J^{\prime(i)}
$$

where $F_{a b}^{(i)}\left(A^{\prime}\right)$ is the field strength for the gauge fields $A_{a}^{\prime(i)}$. For the first two equations, the most general solution is given in terms of the configurations with $A_{v}^{\prime(i)}$ as pure gauge: $A_{v}^{\prime(i)}=$ $-\frac{1}{2} \epsilon^{i j k}\left(\mathcal{O} \partial_{v} \mathcal{O}^{T}\right)^{j k}$, where $\mathcal{O}$ is an arbitrary $3 \times 3$ orthogonal matrix, $\mathcal{O} \mathcal{O}^{T}=\mathcal{O}^{T} \mathcal{O}=1$ with $\operatorname{det} \mathcal{O}=1$. The other gauge field components are given in terms of $v$-independent $S U(2)$ gauge potentials $B_{\theta}^{\prime(i)}$ and $B_{\phi}^{\prime(i)}$ as $A_{\hat{a}}^{\prime(i)}=\mathcal{O}^{i j} B_{\hat{a}}^{(j)}-\frac{1}{2} \epsilon^{i j k}\left(\mathcal{O} \partial_{\hat{a}} \mathcal{O}^{T}\right)^{j k}$ for $\hat{a}=(\theta, \phi)$. Then, since the first two equations of (2.16) are identically satisfied, we are left with the last equation to study:

$$
\frac{k}{2 \pi} F_{\theta \phi}^{(i)}\left(A^{\prime}\right)=\frac{k}{2 \pi} \mathcal{O}^{i j} F_{\theta \phi}^{(j)}\left(B^{\prime}\right) \hat{=}-J^{\prime(i)},
$$

where $F_{\theta \phi}^{(i)}\left(B^{\prime}\right)$ is the $S U(2)$ field strength constructed from gauge fields $\left(B_{\theta}^{\prime(i)}, B_{\phi}^{\prime(i)}\right)$. For this set of gauge configurations, part of the $S U(2)$ gauge invariance has been fixed and, on the spatial slice $\mathbb{S}^{2}$ of $\Delta$, we are now left with invariance only under $v$-independent $S U(2)$ gauge transformations of the fields $B_{\theta}^{\prime(i)}(\theta, \phi)$ and $B_{\phi}^{\prime(i)}(\theta, \phi)$. Next step in this construction is to use this gauge freedom to rotate the triplet $F_{\theta \phi}^{(i)}\left(B^{\prime}\right)$ to a new field strength $F_{\theta \phi}^{(i)}(B)$ parallel to an internal space unit vector $u^{i}(\theta, \phi)$. This can always be achieved through a $v$-independent gauge transformation $\overline{\mathcal{O}}^{i j}(\theta, \phi)$ with components $\overline{\mathcal{O}}^{i 1}(\theta, \phi) \equiv u^{i}(\theta, \phi)$ :

$$
\begin{aligned}
& F_{\theta \phi}^{(i)}\left(B^{\prime}\right)=\overline{\mathcal{O}}^{i j} F_{\theta \phi}^{(j)}(B) \equiv u^{i}(\theta, \phi) F_{\theta \phi}^{(1)}(B), \\
& F_{\theta \phi}^{(1)}(B) \equiv u^{i} F_{\theta \phi}^{(i)}\left(B^{\prime}\right) \neq 0, \quad F_{\theta \phi}^{(2)}(B)=0, \quad F_{\theta \phi}^{(3)}(B)=0,
\end{aligned}
$$

where the primed and unprimed $B$ gauge fields are related by a gauge transformation as: $B_{\hat{a}}^{(i)}=$ $\overline{\mathcal{O}}^{i j} B_{\hat{a}}^{(j)}-\frac{1}{2} \epsilon^{i j k}\left(\overline{\mathcal{O}} \partial_{\hat{a}} \overline{\mathcal{O}}^{T}\right)^{j k}$. We now need to look for the gauge fields $B_{\hat{a}}^{(i)}$ that solve the equations (2.18). There are two types of solutions to these equations. These have been worked out explicitly in the Appendix of [3]. We shall summarize the results in the following.

We may parametrize the internal space unit vector $u^{i}(\theta, \phi)$ in terms of two angles $\Theta(\theta, \phi)$ and $\Phi(\theta, \phi)$ as $u^{i}(\theta, \phi)=\overline{\mathcal{O}}^{i 1}=(\cos \Theta, \sin \Theta \cos \Phi, \sin \Theta \sin \Phi)$. Other components of the orthogonal matrix $\overline{\mathcal{O}}$ in (2.18) may be written as: $\overline{\mathcal{O}}^{i 2}=\cos \chi s^{i}+\sin \chi t^{i}$ and $\overline{\mathcal{O}}^{i 3}=-\sin \chi s^{i}+\cos \chi t^{i}$ where $\chi(\theta, \phi)$ is an arbitrary angle and $s^{i}(\theta, \phi)=(-\sin \Theta, \cos \Theta \cos \Phi, \cos \Theta \sin \Phi), t^{i}(\theta, \phi)=$ $(0,-\sin \Phi, \cos \Phi)$. The angle fields $\Theta(\theta, \phi), \Phi(\theta, \phi)$ and $\chi(\theta, \phi)$ represent the three independent parameters of the uni-modular orthogonal transformation matrix $\overline{\mathcal{O}}$.

Next we express the gauge fields $B_{\hat{a}}^{\prime(i)}$, without any loss of generality, in terms of their components along and orthogonal to the unit vector $u^{i}$ as:

$$
B_{\hat{a}}^{(i)}=u^{i} B_{\hat{a}}+f \partial_{\hat{a}} u^{i}+g \epsilon^{i j k} u^{j} \partial_{\hat{a}} u^{k}, \quad \hat{a}=(\theta, \phi)
$$

with the field strength constructed from these as:

$$
\begin{aligned}
F_{\hat{a} \hat{b}}^{(i)}\left(B^{\prime}\right)= & u^{i}\left(2 \partial_{[\hat{a}} B_{\hat{b}]}+\left(f^{2}+g^{2}+2 g\right) \epsilon^{j k l} u^{j} \partial_{\hat{a}} u^{k} \partial_{\hat{b}} u^{l}\right) \\
& +2 \partial_{[\hat{a}} u^{i}\left((1+g) B_{\hat{b}]}-\partial_{\hat{b}]} f\right)-2 \epsilon^{i j k} u^{j} \partial_{[\hat{a}} u^{k}\left(f B_{\hat{b}]}+\partial_{\hat{b}]} g\right) .
\end{aligned}
$$


Six independent field degrees of freedom in $B_{\hat{a}}^{(i)}$ are now distributed in $u^{i}$ (two independent fields), $B_{\hat{a}}$ (two field degrees of freedom) and two fields $(f, g)$.

Requiring the field strength $F_{\hat{a} \hat{b}}^{(i)}\left(B^{\prime}\right)$ in (2.19) to satisfy the equations (2.18), gives us equations for various component fields $f, g$ and $B_{\hat{a}}$ which we need to solve. There are two possible solutions to the equations so obtained. These can be expressed through two types of gauge fields $B_{\hat{a}}^{(i)}$. These gauge fields are related to $B_{\hat{a}}^{(i)}$ through gauge transformation $\overline{\mathcal{O}}$ as indicated in (2.18). We just list these two solutions here: (a) The first solution is given by: $f=0, g=-1$ with $B_{\hat{a}}$ as arbitrary, leading to $B_{\hat{a}}^{(i)}=\left(B_{\hat{a}}+\cos \Theta \partial_{\hat{a}} \Phi, 0,0\right)$. Now the configuration (2.7) with its field strength as in (2.8) above can be identified with this solution for $B_{\hat{a}}=0$ and $\Theta=\theta, \Phi=\phi$ and coupling $k=A_{\mathrm{H}} /(4 \gamma)$. (b) The second solution is given by $f=c \cos \delta$, $1+g=c \sin \delta$ and $B_{\hat{a}}=-\partial_{\hat{a}} \delta$ with $c$ as a constant and $\delta(\theta, \phi)$ arbitrary. This leads to the gauge configuration: $B_{\hat{a}}^{(1)}=-\partial_{\hat{a}} \delta+\cos \Theta \partial_{\hat{a}} \Phi, B_{\hat{a}}^{(2)}=c\left(\cos \delta \partial_{\hat{a}} \Theta-\sin \delta \sin \Theta \partial_{\hat{a}} \Phi\right)$ and $B_{\hat{a}}^{(3)}=c\left(\sin \delta \partial_{\hat{a}} \Theta+\cos \delta \sin \Theta \partial_{\hat{a}} \Phi\right)$. Now the configuration (2.10) with its field strength components satisfying the equations (2.11) can be identified with this solution for $c=-K$ and $\Theta=\theta, \Phi=\phi$ and $\delta$ as a constant. Further, for $c=0$ and constant $\delta$, this solution coincides with the first solution (a) above for $B_{\hat{a}}=0$.

Finally, we may rewrite the starting $S U(2)$ gauge configurations $A_{a}^{\prime i}$ of (2.16), for both these cases, as: $A_{v}^{\prime(i)}=-\frac{1}{2} \epsilon^{i j k}\left(\mathcal{O}^{\prime} \partial_{v} \mathcal{O}^{\prime T}\right)^{j k}, A_{\hat{a}}^{\prime(i)}=\mathcal{O}^{\prime i j} B_{\hat{a}}^{(j)}-\frac{1}{2} \epsilon^{i j k}\left(\mathcal{O}^{\prime} \partial_{\hat{a}} \mathcal{O}^{\prime T}\right)^{j k}$ where $\mathcal{O}^{\prime}$ is the product of gauge transformation matrices introduced in (2.17) and (2.18): $\mathcal{O}^{\prime}=\mathcal{O} \overline{\mathcal{O}}$. The first two equations of (2.16) are identically satisfied and the last equation becomes

$$
\frac{k}{2 \pi} F_{\theta \phi}^{(i)}\left(A^{\prime}\right)=\frac{k}{2 \pi} \mathcal{O}^{\prime i j} F_{\theta \phi}^{(j)}(B) \hat{=}-J^{\prime(i)} \equiv-\mathcal{O}^{\prime i j} J^{(j)}
$$

where now from $(2.18), F_{\theta \phi}^{(i)}(B)=\left(F_{\theta \phi}^{(1)}(B), 0,0\right)$, which implies for the sources $J^{(i)}=(J, 0,0)$. Thus, this gauge fixing procedure leads to a theory described in terms of fields $B_{\hat{a}}^{(i)}$ with a left over invariance only under $U(1)$ gauge transformations.

This completes our discussion of how the horizon properties can be described by a $S U(2)$ Chern-Simons gauge theory or equivalently, by a gauge fixed version with only $U(1)$ invariance.

\subsection{Isolated horizons}

In order to define horizons in a manner decoupled from the bulk, a generalized notion of Isolated Horizon ( $\mathrm{IH})$, as a quasi-local replacement of the event horizon of a black hole, has been developed by Ashtekar et al. [2]. This is done by ascribing attributes which are defined on the horizon intrinsically through a set of quasi-local boundary conditions without reference to any assumptions like stationarity such that the horizon is isolated in a precise sense. This permits us to describe a black hole in equilibrium with a dynamical exterior region. An IH is defined to be a null surface, with topology $\mathbb{S}^{2} \times \mathbb{R}$, which is non-expanding and shear-free. The various geometric quantities on such a horizon are seen to satisfy $U(1)$ Chern-Simons equations of motion [9]:

$$
F_{r \theta}=0, \quad F_{r \phi}=0, \quad F_{\theta \phi}=-\frac{2 \pi}{k} \Sigma_{\theta \phi}^{(1)}
$$

where $r, \theta$ and $\phi$ are the coordinates on the horizon and $k=A_{\mathrm{H}} /(4 \gamma)$ with $A_{\mathrm{H}}$ as the horizon area, $\gamma$ is the Barbero-Immirzi parameter, $F_{a b}$ is the field strength of $U(1)$ gauge field. The source $\Sigma_{\theta \phi}^{(1)}$ is one component of the $S U(2)$ triplet of solder forms $\Sigma_{\theta \phi}^{(i)} \equiv \gamma^{-1} \epsilon^{i j k} e_{\theta}^{j} e_{\phi}^{k}$, in the direction of the subgroup $U(1)$. There is another fact which is not some times stated explicitly. The horizon boundary conditions, which lead to the equations (2.20), also further imply 
the following constraints for the components of the triplet of solder forms in the internal space directions orthogonal to the $U(1)$ direction:

$$
\Sigma_{\theta \phi}^{(2)}=0, \quad \Sigma_{\theta \phi}^{(3)}=0
$$

Now this is exactly the same situation as we came across for the Schwarzschild hole in Section 2.1 above. Just like there, the equations (2.20) and (2.21) really describe a $S U(2)$ Chern-Simons theory partially gauge fixed to leave only a leftover $U(1)$ invariance.

Thus, as in the case of the Schwarzschild hole, the degrees of freedom of the more general Isolated Horizon are also described by a quantum $S U(2)$ Chern-Simons gauge theory with specific sources given in terms of the solder fields. An equivalent description is provided by a gauge fixed version of this theory in terms of the quantum $U(1)$ Chern-Simons theory represented by the operator constraint (2.20), but with the physical states satisfying additional conditions which are the quantum analogues of the classical constraints (2.21). Horizon properties like the entropy can be calculated in either version with the same consequences. We shall review these calculations in the following.

\section{Micro-canonical entropy}

Over last several decades, many authors have developed methods based on $S U(2)$ gauge theory to count the micro-states associated with a two-dimensional surface. Smolin was first to explore the use of $S U(2)$ Chern-Simons theory induced on a boundary satisfying self-dual boundary conditions in Euclidean gravity [10]. He also demonstrated that such a boundary theory obeys the Bekenstein bound. Krasnov applied these ideas to the black hole horizon and used the ensemble of quantum states of $S U(2)$ Chern-Simons theory associated with the spin assignments of the punctures on the surface to count the boundary degrees of freedom and reproduced an area law for the entropy [11]. In this first application of $S U(2)$ Chern-Simons theory to black hole entropy, the gauge coupling was taken to be proportional to the horizon area and also inversely proportional to the Barbero-Immirzi parameter. Assuming that the quantum states of a fluctuating black hole horizon to be governed by the properties of intersections of knots carrying $S U(2)$ spins with the two-dimensional surface, Rovelli also developed a counting procedure which again yielded an area law for the entropy [12]. In the general context of Isolated Horizons, it was the pioneering work of Ashtekar, Baez, Corichi and Krasnov [9] which studied $S U(2)$ Chern-Simons theory as the boundary theory and the area law for entropy was again reproduced. This was further developed in $[13,14,15,16]$ which extensively exploited the deep connection between the three dimensional Chern-Simons theory and the conformal field theories in two dimensions. This framework provided a method to calculate corrections beyond the area law for micro-canonical entropy of large black holes. In particular, it is more than ten years now when a leading correction given by the logarithm of horizon area with a definite coefficient of $-3 / 2$ followed by sub-leading terms containing a constant and inverse powers of area were first obtained [14]:

$$
S_{\mathrm{bh}}=S_{\mathrm{BH}}-\frac{3}{2} \ln S_{\mathrm{BH}}+\text { const }+\mathcal{O}\left(S_{\mathrm{BH}}^{-1}\right),
$$

where $S_{\mathrm{BH}}=A_{\mathrm{H}} /\left(4 \ell_{P}^{2}\right)$ is the Bekenstein-Hawking entropy given in terms of horizon area $A_{\mathrm{H}}$. The corrections due to the non-perturbative effects represented by the discrete quantum geometry are finite. These may be contrasted with those obtained in Euclidean path integral formulation from the graviton and other quantum matter fluctuations around the hole back ground which depend on the renormalization scale [17]. 


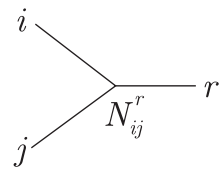

$(a)$

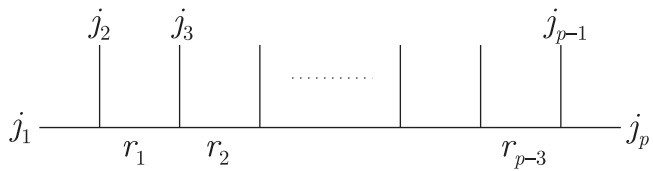

(b)

Figure 1. Diagrammatic representation for (a) the fussion matrix $N_{i j}^{r}$ and (b) for the composition rule (3.1) for spins $j_{1}, j_{2}, j_{3}, \ldots, j_{p}$.

\subsection{Horizon entropy from the $S U(2)$ Chern-Simons theory}

In this subsection, we shall survey the general framework developed in $[13,14]$ for studying the horizon properties in the $S U(2)$ Chern-Simons formulation. An important ingredient in counting the horizon micro-states is the fact $[18,19]$ that Chern-Simons theory on a three-manifold with boundary can be completely described by the properties of a gauged Wess-Zumino conformal theory on that two dimensional boundary. Starting with the pioneering work of Witten leading to Jones polynomials [18], this relationship has been extensively used to study Chern-Simons theories. This includes methods to solve the Chern-Simons theories explicitly and exactly and also to construct three-manifold invariants from generalized knot/link invariants in these theories [20].

In the LQG, the Hilbert space of canonical quantum gravity is described by spin networks with Wilson line operators carrying $S U(2)$ representations ( the edges of the graph. Sources of the boundary $S U(2)$ Chern-Simons theory (with coupling $k=A_{\mathrm{H}} /(4 \gamma)$ ) describing the horizon properties are given in terms of the solder forms which are quantum fields of the bulk theory. These have distributional support at the punctures at which the bulk spin network edges impinge on the horizon. Given the relationship of ChernSimons theory and the two dimensional conformal field theory mentioned above, the Hilbert space of states of $S U(2)$ Chern-Simons theory with coupling $k$ on a three-manifold $\mathbb{S}^{2} \times \mathbb{R}$ (horizon) is completely characterized by the conformal blocks of the $S U(2)_{k}$ Wess-Zumino conformal theory on an $\mathbb{S}^{2}$ with punctures $\mathcal{P} \equiv\{1,2, \ldots, p\}$ where each puncture carries spin $j_{i}=1 / 2,1,3 / 2, \ldots, k / 2$.

$S U(2)_{k}$ conformal field theory is described in terms of primary fields $\phi_{j}$ with spin values cut off by the maximum value $k / 2, j=0,1 / 2,1, \ldots, k / 2$. The composition rule for two spin $j$ and $j^{\prime}$ representations is modified from that in the corresponding ordinary $S U(2)$ as: $(j) \otimes\left(j^{\prime}\right)=(\mid j-$ $\left.j^{\prime} \mid\right) \oplus\left(\left|j-j^{\prime}\right|+1\right) \oplus\left(\left|j-j^{\prime}\right|+2\right) \oplus \cdots \oplus\left(\min \left(j+j^{\prime}, k-j-j^{\prime}\right)\right)$. We may rewrite this composition law for the primary fields $\left[\phi_{i}\right]$ and $\left[\phi_{j}\right]$ as: $\left[\phi_{i}\right] \otimes\left[\phi_{j}\right]=\sum_{r} N_{i j}^{r}\left[\phi_{r}\right]$ in terms of the fusion matrices $N_{i j}^{r}$ whose elements have values 1 or 0 , depending on whether the primary field $\left[\phi_{r}\right]$ is allowed or not in the product. Representing the fusion matrix $N_{i j}^{r}$ diagrammatically as in Fig. 1(a), the composition of $p$ primary fields in spin representations $j_{1}, j_{2}, j_{3}, \ldots, j_{p}$ can be depicted by the diagram in Fig. 1(b). Then the total number of conformal blocks with spin $j_{1}, j_{2}, \ldots, j_{p}$ on the external lines (associated with the $p$ punctures on $\mathbb{S}^{2}$ ) and spins $r_{1}, r_{2}, \ldots, r_{p-3}$ on the internal lines in this composition diagram is the product of $(p-2)$ factors of fusion matrix as given by:

$$
\mathcal{N}_{\mathcal{P}}=\sum_{\left\{r_{i}\right\}} N_{j_{1} j_{2}}^{r_{1}} N_{r_{1} j_{3}}^{r_{2}} N_{r_{2} j_{4}}^{r_{3}} \cdots N_{r_{p-3} j_{p-1}}^{j_{p}}
$$

There is a remarkable result due to Verlinde which states that the fusion matrices $\left(N_{i}\right)_{j}^{r} \equiv$ $N_{i j}^{r}$ of a conformal field theory are diagonalised by the unitary duality matrices $S$ associated with modular transformation $\tau \rightarrow-1 / \tau$ of the torus. This fact immediately leads to the Verlinde formula which expresses the components of the fusion matrix in terms of those of 
this $S$ matrix [21]:

$$
N_{i j}^{r}=\sum_{s} \frac{S_{i s} S_{j s} S_{s}^{\dagger r}}{S_{0 s}} .
$$

For the $S U(2)_{k}$ Wess-Zumino conformal theory, the duality matrix $S$ is explicitly given by

$$
S_{i j}=\sqrt{\frac{2}{k+2}} \sin \left(\frac{(2 i+1)(2 j+1) \pi}{k+2}\right),
$$

where $i=0,1 / 2,1, \ldots, k / 2$ and $j=0,1 / 2,1, \ldots, k / 2$ are the spin labels.

The fusion rules and Verlinde formula above were first obtained in the conformal theory context. It is also possible to derive these results directly in the Chern-Simons theory, using only the gauge theory techniques without taking recourse to the conformal field theory. This has been done in the paper of Blau and Thompson in [19]. This paper also discusses how the Chern-Simons theory based on a compact gauge group $G$ can be abelianized to a topological field theory based on the maximal torus $T$ of $G$. In particular, for the $S U(2)$ Chern-Simons theory, this framework describes the gauge fixing to the maximal torus of $S U(2)$ which is its $U(1)$ subgroup.

Now, the formula (3.1) for the number of conformal blocks $\mathcal{N}_{\mathcal{P}}$ for the set of punctures $\mathcal{P}$ can be rewritten, using (3.2) and unitarity of the matrix $S$, as:

$$
\mathcal{N}_{\mathcal{P}}=\sum_{r=0}^{k / 2} \frac{S_{j_{1} r} S_{j_{2} r} \cdots S_{j_{p} r}}{\left(S_{0 r}\right)^{p-2}}
$$

which further, using the explicit formula for the duality matrix $(3.3)$, leads to $[19,21,13,14]$ :

$$
\mathcal{N}_{\mathcal{P}}=\frac{2}{k+2} \sum_{r=0}^{k / 2} \frac{\prod_{l=1}^{p} \sin \left(\frac{\left(2 j_{l}+1\right)(2 r+1) \pi}{k+2}\right)}{\left[\sin \left(\frac{(2 r+1) \pi}{k+2}\right)\right]^{p-2}} .
$$

This master formula just counts the number of ways $p$ primary fields in spin $j_{1}, j_{2}, \ldots, j_{p}$ representations associated with the $p$ punctures on $\mathbb{S}^{2}$ of horizon can be composed into $S U(2)$ singlets. Notice the presence of combination $k+2$ in this formula. This just reflects the fact that the effective coupling constant of quantum $S U(2)$ Chern-Simons theory is $k+2$ instead of its classical value $k$.

Now, the horizon entropy is given by counting the micro-states by summing $\mathcal{N}_{\mathcal{P}}$ over all possible sets of punctures and then taking its logarithm:

$$
\mathcal{N}_{\mathrm{H}}=\sum_{\{\mathcal{P}\}} \mathcal{N}_{\mathcal{P}}, \quad S_{\mathrm{H}}=\ln \mathcal{N}_{\mathrm{H}}
$$

for a fixed horizon area $A_{\mathrm{H}}$ (or more accurately with nearby area values in a sufficiently narrow range with this fixed mid point value). In LQG, area for a punctured $\mathbb{S}^{2}$, with the spins $j_{1}, j_{2}, \ldots, j_{p}$ on the $p$ punctures is given by [6]:

$$
A_{\mathrm{H}}=8 \pi \gamma \sum_{l=1,2, \ldots, p} \sqrt{j_{l}\left(j_{l}+1\right)}
$$

in the units where the Planck length $\ell_{P}=1$. Here $\gamma$ is the Barbero-Immirzi parameter. 
A straightforward reorganization of the master formula (3.4), through a redefinition of the dummy variables and using the fact that the product in this formula can be written as a multiple sum, leads to an alternative equivalent expression as [13]:

$$
\mathcal{N}_{\mathcal{P}}=\frac{2}{k+2} \sum_{\ell=1,2, \ldots}^{k+1} \sin ^{2} \frac{\theta_{\ell}}{2}\left\{\sum_{m_{1}=-j_{1}}^{j_{1}} \cdots \sum_{m_{p}=-j_{p}}^{j_{p}} \exp \left[i \theta_{\ell}\left(m_{1}+m_{2}+\cdots+m_{p}\right)\right]\right\}
$$

with $\theta_{\ell} \equiv \frac{2 \pi l}{k+2}$. Now, we use the representation for a periodic Kronecker delta, with period $k+2$ :

$$
\bar{\delta}_{m_{1}+m_{2}+\cdots+m_{p}, m} \equiv \frac{1}{k+2} \sum_{\ell=0}^{k+1} \exp \left[i \theta_{\ell}\left(m_{1}+m_{2}+\cdots+m_{p}-m\right)\right]
$$

Expanding the $\sin ^{2} \frac{\theta_{\ell}}{2}$ factor in the formula (3.7) and after an interchange of the summations, this formula can be recast as [13]:

$$
\mathcal{N}_{\mathcal{P}}=\sum_{m_{1}=-j_{1}}^{j_{1}} \cdots \sum_{m_{p}=-j_{p}}^{j_{p}}\left(\bar{\delta}_{m_{1}+m_{2}+\cdots+m_{p}, 0}-\frac{1}{2} \bar{\delta}_{m_{1}+m_{2}+\cdots+m_{p}, 1}-\frac{1}{2} \bar{\delta}_{m_{1}+m_{2}+\cdots+m_{p},-1}\right) .
$$

The various terms here have specific special interpretations [15]: The first term just counts the total number of ways the 'magnetic' quantum number $m$ of the spin $j_{1}, j_{2}, \ldots, j_{p}$ assignments on the $p$ punctures can be added to yield total $m_{\text {tot }}=\sum_{l=1}^{p} m_{l}=0$ modulo $k+2$. This sum over counts the total number of singlets $\left(j_{\text {tot }}=0\right)$ in the composition of primary fields with spins $j_{1}, j_{2}, \ldots, j_{p}$, because it also includes those states with $m_{\text {tot }}=0$ coming from configurations with total spin $j_{\text {tot }}=1,2, \ldots$ in the product representation $\otimes_{l=1}^{p}\left(j_{l}\right) \equiv\left(j_{1}\right) \otimes\left(j_{2}\right) \otimes \cdots \otimes\left(j_{p}\right)$. Such states are always accompanied by those with $m_{\text {tot }}= \pm 1$ in the product $\otimes_{l=1}^{p}\left(j_{l}\right)$. Hence these can be counted by enumerating the number of ways the $m$ quantum numbers of the spin representations $j_{1}, j_{2}, \ldots, j_{p}$ add up to $m_{\text {tot }}=\sum_{l}^{p} m_{l}=+1$ (modulo $\left.k+2\right)$ or $m_{\text {tot }}=-1$ (modulo $k+2)$. Note, these two numbers are equal which makes the last two terms in (3.8) equal. Hence with the normalization factor $1 / 2$ in each of them, these two terms precisely subtract the number of extra states so that formula (3.8) counts exactly the number of singlet states in the product $\otimes_{l=1}^{p}\left(j_{l}\right)$

Presence of the periodic Kronecker deltas $\bar{\delta}_{m, n}$ in (3.8) distinguishes this formula of the $S U(2)_{k}$ Wess-Zumino conformal field theory from the corresponding group theory formula for $S U(2)$ with ordinary Kronecker deltas $\delta_{m, n}$. In the large limit $k(k \gg 1)$, the periodic Kronecker delta $\bar{\delta}_{m, n}$ can be approximated by the ordinary Kronecker delta $\delta_{m, n}$; hence in this limit, the equation (3.8) leads to the ordinary $S U(2)$ group theoretic formula for counting singlets in the composite representation $\otimes_{l=1}^{p}\left(j_{l}\right)$.

The master formula (3.4) along with its equivalent representations (3.7) and (3.8) and the entropy formula (3.5) are exact and provide a general framework, first set up in [13, 14], for study of horizon entropy. For large horizons, suitable approximate methods have been adopted to extract interesting results from these equations. For fixed large values of $p$ and the horizon area $A_{\mathrm{H}}$, it is clear that the largest contribution to the degeneracy of horizon states will come from low values of the spins $j_{i}$ assigned to the punctures. For computational simplicity, let us put spin $1 / 2$ representations on all the puncture sites on $\mathbb{S}^{2}$. The dimension of the associated Hilbert space in this case can be readily evaluated. To obtain the leading behaviour for large $k$ $\left(=\frac{A_{\mathrm{H}}}{4 \gamma}\right)$, the state counting can as well be done using ordinary $S U(2)$ rules. It is straight 
forward to check that, for the case with spin $1 / 2$ on all the punctures, this yields:

$$
\mathcal{N}_{\mathcal{P}}=\left(\begin{array}{c}
p \\
p / 2
\end{array}\right)-\left(\begin{array}{c}
p \\
(p / 2-1)
\end{array}\right)
$$

The first term here follows from the first term of (3.8) in the limit of large $k(k \gg 1)$ and simply counts the number of ways $m_{i}= \pm 1 / 2$ assignments can be put on $p$ (even) punctures such that $\sum m_{i}=0$. The second term of (3.9) similarly follows from the second and third terms of (3.8), counting the number of ways assignments $m_{i}= \pm 1 / 2$ are placed on the punctures such that their sum is +1 or -1 . The difference of these two terms counts the number of $S U(2)$ singlets in the product of $p$ spin $1 / 2$ representations. The expression (3.9) is also equal to $n$th number in the Catalan series $C_{n}=\frac{(2 n) !}{(n+1) ! n !}$ for $p=2 n$. For large $p$, using Stirling formula, this leads to $[14,15]$ :

$$
\mathcal{N}_{\mathcal{P}}=C \frac{2^{p}}{p^{3 / 2}}\left\{1+\mathcal{O}\left(\frac{1}{p}\right)\right\}
$$

where $C$ is a $p$ independent numerical constant ${ }^{2}$. Instead, if we place spin 1 on all the $p$ punctures, this number is [16]: $\mathcal{N}_{\mathcal{P}}=C \frac{3^{p}}{p^{3 / 2}}\left\{1+\mathcal{O}\left(\frac{1}{p}\right)\right\}$. More generally, for the case where all the punctures carry the same low spin value $r(r \ll p$ and $r \ll k)[16]$, we have ${ }^{3}$ :

$$
\mathcal{N}_{\mathcal{P}}=C \frac{(2 r+1)^{p}}{p^{3 / 2}}\left\{1+\mathcal{O}\left(\frac{1}{p}\right)\right\}
$$

The corresponding entropy for all these cases is:

$$
S_{\mathrm{H}}=\ln \mathcal{N}_{\mathcal{P}}=p \ln (2 r+1)-\frac{3}{2} \ln p+\mathcal{O}\left(p^{0}\right) .
$$

Now, the area of a two-surface with Wilson lines, carrying common spin $r$ representation, impinging on it at $p$ punctures, is given by (3.6) as: $A_{\mathrm{H}}=8 \pi \gamma p \sqrt{r(r+1)}$. Inverting this to write $p$ in terms of the horizon area $A_{\mathrm{H}}, p=A_{\mathrm{H}}[8 \pi \gamma \sqrt{r(r+1)}]^{-1}$, leads to the entropy formula for large area as [14]:

$$
S_{\mathrm{H}}=\frac{A_{\mathrm{H}}}{4}-\frac{3}{2} \ln \left(\frac{A_{\mathrm{H}}}{4}\right)+\text { const }+\mathcal{O}\left(A_{\mathrm{H}}^{-1}\right),
$$

\footnotetext{
${ }^{2}$ It is also possible to count the number $\mathcal{N}_{\mathcal{P}}^{(j)}$ of ways spin $1 / 2$ representations on $p$ punctures can be composed to yield, instead of the singlets, net spin $j$ representations. This follows from straight forward application of the techniques developed in [14] which yield, for $j \ll k$ and large $k$ and $p: \mathcal{N}_{\mathcal{P}}^{(j)} \sim 2^{p+2}[F(p)-F(p+2)]$ where $F(p) \approx \frac{1}{\pi} \int_{0}^{\pi} d x\left(\frac{\sin [(2 j+1) x]}{\sin x}\right) \cos ^{p} x$. For $j \ll p$, this integral can be evaluated to be $F(p) \sim \frac{(2 j+1)}{\sqrt{p}}\left\{1+\mathcal{O}\left(\frac{1}{p}\right)\right\}$ which finally leads to the formula: $\mathcal{N}_{\mathcal{P}}^{(j)} \sim \frac{(2 j+1) 2^{p}}{p^{3 / 2}}\left\{1+\mathcal{O}\left(\frac{1}{p}\right)\right\}$ [Kaul R.K., Kalyana Rama S., unpublished]. The angular momentum of a rotating black hole is to be defined with respect to the global rotation properties of the space-time at spatial infinity. In the LQG, internal gauge group $S U(2)$ is asymptotically linked with these global rotations leading to an identification of angular momentum with internal spin at this boundary. However, from the point of view of the horizon boundary theory, angular momentum, like all other properties of the black hole, has to be described completely in terms of the horizon attributes which are codified in the topological properties of the punctures carrying the internal spins $\vec{J}_{i}$ on them. Such an angular momentum operator has to obey the standard $S O(3)$ algebra $\left[J^{(l)}, J^{(m)}\right]=i \epsilon^{l m n} J^{(n)}$. An operator with these properties is the total spin $\vec{J}=\sum_{i=1}^{p} \vec{J}_{i}$. This perspective, therefore, suggests that $\mathcal{N}_{\mathcal{P}}^{(j)}$ above represents the degeneracy associated with the horizon states of a rotating hole with quantum angular momentum given by the eigenvalues $j(j+1)$ of $\vec{J}$. $\vec{J}$ and its logarithm may be interpreted as the entropy.

${ }^{3}$ Note that for $r$ with half-integer values, the number of punctures $p$ has to be even in order to get a non-zero number of net $j_{\text {tot }}=0$ states.
} 
where the Barbero-Immirzi parameter is fixed to be $\gamma=\frac{\ln (2 r+1)}{2 \pi \sqrt{r(r+1)}}$ to match the linear area term with the Bekenstein-Hawking law. The linear area term for $r=1 / 2$ had been already obtained in [9]. The framework of $[13,14]$ provides a systematic procedure of deriving corrections beyond this leading term. An important point to note here is that the sub-leading logarithmic correction is independent of $\gamma$ and is insensitive to the value of spin $r(r \neq 0)$ placed on the punctures. But, the coefficient of leading area term does depend on spin value $r$ and hence $\gamma$ changes as we change $r$. The general form of the formula (3.10) is robust enough, though the coefficient of the linear area term and thereby the value of the Barbero-Immirzi parameter are not.

We shall close this discussion with one last remark. The derivation of the leading terms of the asymptotic entropy formula (3.10) does not require the full force of the conformal field theory. It was already pointed out in $[15,16]$ and as has been seen above that, instead of full $S U(2)_{k}$ composition rules, use of ordinary $S U(2)$ rules (corresponding to large $k\left(=\frac{A_{\mathrm{H}}}{4 \gamma}\right)$ limit) is good enough for the relevant counting to yield the first two terms of entropy formula (3.10). These results have been re-derived in [22] where ordinary $S U(2)$ composition over the spins is computed through an equivalent representation in terms of a random walk modified with a mirror at origin. In this study, the coefficient $-3 / 2$ for the logarithmic correction is again confirmed and this coefficient is interpreted as a reflection of entanglement between parts of the horizon. However, the terms beyond the logarithmic correction are sensitive to the details of conformal theory resulting in effects which are more pronounced for smaller $k$. Thus two ways of counting would show differences in these terms.

\subsection{Improved value of the Barbero-Immirzi parameter}

Early calculations of the entropy, as discussed above, were done by taking a common low value of spin, $1 / 2$ or $1, \ldots$, on all the punctures. Soon it was realized that this approximation needs to be improved [23]. In fact, maximized entropy, subject to holding the horizon area fixed within a sufficiently narrow range $\left[A_{\mathrm{H}}-\epsilon, A_{\mathrm{H}}+\epsilon\right]$, is obtained for configurations with different spin values $1 / 2,1,3 / 2,2, \ldots$ distributed over the punctures in a definite way. The relevant configurations are those where fraction $f_{j}$ of $p$ punctures carrying spin $j$ representation is given by the probability distribution [23, 24]:

$$
f_{j} \equiv \frac{n_{j}}{p}=(2 j+1) \exp (-\lambda \sqrt{j(j+1)}) .
$$

From this, we have

$$
\sum_{j=1 / 2}^{\infty} f_{j} \equiv \sum_{j=1 / 2}^{\infty}(2 j+1) \exp (-\lambda \sqrt{j(j+1)})=1,
$$

which when solved numerically yields $\lambda \approx 1.72$. Using this value of $\lambda$, the distribution (3.11) implies that the configurations of interest contain fractions $f_{j} \approx 0.45,0.26,0.14,0.07,0.04,0.02, \ldots$ of total number of $p$ punctures with spins $j=1 / 2,1,3 / 2,2,5 / 2,3, \ldots$ respectively. Notice that the low spin values have higher occupancies; those for higher spin fall off rapidly.

This improved counting of net $S U(2)$ spin zero configurations does not change the general form of the asymptotic entropy formula (3.10). In particular, as we shall see below, the coefficient $-3 / 2$ of the logarithmic term is unaltered ${ }^{4}$. Only change is in the coefficient of the leading area term.

\footnotetext{
${ }^{4}$ The computations in $[23,24]$ were originally done in the $U(1)$ framework without imposing the quantum analogues of the additional constraints (2.21) and logarithmic correction turned out to be with a coefficient $-1 / 2$. Same $-\left(\ln A_{\mathrm{H}}\right) / 2$ correction was earlier obtained through the counting rules analogous to those in $U(1)$ theory in [15]. However, when done with care including these additional constraints, this coefficient gets corrected to $-3 / 2$. See the discussion in Section 3.3.
} 
The equations (3.11) and (3.12) have been derived in [23, 24] by maximizing the entropy subject to the fixed area constraint without any further conditions so that at each puncture carrying spin $j$, there are $2 j+1$ possible degrees of freedom. Imposing further constraints, in the $U(1)$ formulation, so that the net $U(1)$ charge on all the punctures is zero, modifies these equations only marginally [24]. For the case of $S U(2)$ formulation where the spins on all the punctures have to add up to form $S U(2)$ singlets, the corresponding equations also have modifications which, as we shall see below, are suppressed as powers of inverse area.

In the $S U(2)$ Chern-Simons formulation, for a configuration where spin $j$ lives on $n_{j}$ punctures, the degeneracy formula (3.4) for the set of punctures $\{\mathcal{P}\}$ with occupancy numbers $\left\{n_{j}\right\}$ can be recast as:

$$
\mathcal{N}\left(\left\{n_{j}\right\}\right)=\left[\frac{\left(\sum_{j} n_{j}\right) !}{\prod_{j} n_{j} !}\right] \frac{2}{k+2} \sum_{\ell=1,2, \ldots}^{k+1} \sin ^{2} \frac{\theta_{\ell}}{2} \prod_{j}\left[\frac{\sin \left(\frac{(2 j+1) \theta_{\ell}}{2}\right)}{\sin \frac{\theta_{\ell}}{2}}\right]^{n_{j}},
$$

where $\theta_{\ell} \equiv \frac{2 \pi \ell}{k+2}$ and $\sum_{j} n_{j}=p$ is the total number of punctures. The total degeneracy of the horizon states is obtained by summing over all possible values for the occupancies $\left\{n_{j}\right\}$. The pre factor in the right-hand side of above equation comes from the fact that the spin $j$ can be placed on any of the $n_{j}$ sites from the set of all $p$ punctures reflecting the fact that the punctures are distinguishable. Formula (3.13) can be rewritten as:

$$
\mathcal{N}\left(\left\{n_{j}\right\}\right)=\frac{\left(\sum n_{j}\right) !}{\prod n_{j} !}\left[I_{0}\left(\left\{n_{j}\right\}\right)-I_{1}\left(\left\{n_{j}\right\}\right)\right],
$$

where

$$
\begin{aligned}
& I_{0} \equiv \frac{1}{k+2} \sum_{\ell=1,2, \ldots}^{k+1} \prod_{j}\left[\frac{\sin \left(\frac{(2 j+1) \theta_{\ell}}{2}\right)}{\sin \frac{\theta_{\ell}}{2}}\right]^{n_{j}}=\frac{1}{k+2} \sum_{\ell=1,2, \ldots}^{k+1} \prod_{j}\left[\sum_{m=-j}^{j} e^{i m \theta_{\ell}}\right]^{n_{j}}, \\
& I_{1} \equiv \frac{1}{k+2} \sum_{\ell=1,2, \ldots}^{k+1} \cos \theta_{\ell} \prod_{j}\left[\frac{\sin \left(\frac{(2 j+1) \theta_{\ell}}{2}\right)}{\sin \frac{\theta_{\ell}}{2}}\right]^{n_{j}}=\frac{1}{k+2} \sum_{\ell=1,2, \ldots}^{k+1} \cos \theta_{\ell} \prod_{j}\left[\sum_{m=-j}^{j} e^{i m \theta_{\ell}}\right]^{n_{j}} .
\end{aligned}
$$

The quantity $I_{0}-I_{1}$ simply counts the number of possible $S U(2)$ singlets in the product representations of spins $j$ with occupancies $n_{j}$ on the punctures. Here $I_{0}$ counts the numbers of ways the 'magnetic' quantum numbers $m$ can be put on the various punctures so that their sum is zero and $I_{1}$ counts the corresponding number where their sum is +1 or equivalently, the sum is -1 .

The relevant configurations are obtained by maximizing the entropy $S=\ln \mathcal{N}\left(\left\{n_{j}\right\}\right)$ subject to the constraint that the horizon area has a fixed large value $A_{\mathrm{H}}=8 \pi \gamma \sum n_{j} \sqrt{j(j+1)}$ in the $\ell_{P}=1$ units. This is done through solving the maximization condition for $n_{j}$ :

$$
\delta \ln \mathcal{N}\left(\left\{n_{j}\right\}\right)-\frac{\lambda}{8 \pi \gamma} \delta A_{\mathrm{H}}=0,
$$

where $\lambda$ is a Lagrange multiplier. This finally leads to a formula for the horizon entropy. The techniques developed in $[14,15]$ and [24] can be easily extended to perform the computations in a simple and straight forward manner for large area, $A_{\mathrm{H}} \gg 1$. We now outline these calculations.

Using Stirling's formula for the factorial of a large number, equation (3.14) can be solved for large $n_{j}$ to yield:

$$
f_{j} \equiv \frac{n_{j}}{\sum_{j} n_{j}}=\exp \left[-\lambda \sqrt{j(j+1)}+\frac{\delta \ln I}{\delta n_{j}}\right],
$$

where $I \equiv I_{0}\left(\left\{n_{j}\right\}\right)-I_{1}\left(\left\{n_{j}\right\}\right)$. 
For large areas, calculation can be done in the large $k\left(=\frac{A_{\mathrm{H}}}{4 \gamma}\right)$ limit where the summations in $I_{0}$ and $I_{1}$ can be replaced by integrals: $\theta_{\ell}=\frac{2 \pi \ell}{k+2} \rightarrow x$ and $\frac{1}{k+2} \sum_{\ell=1,2, \ldots}^{k+1} \rightarrow \frac{1}{2 \pi} \int_{0}^{2 \pi} d x$. Writing these integrals as: $I_{0} \approx \frac{1}{2 \pi} \int d x \exp [F(x)]$ and $I_{1} \approx \frac{1}{2 \pi} \int d x \exp [F(x)+\ln \cos x]$ with $F(x) \equiv \sum_{j} n_{j}\left[\ln \sin \left((2 j+1) \frac{x}{2}\right)-\ln \sin \frac{x}{2}\right]$, these can be readily evaluated by the steepest descent method. Each of $F(x)$ and $F(x)+\ln \cos x$ has a maximum at $x=0$. Evaluating the integrals by quadratic fluctuations around this maximum point leads to:

$$
\begin{aligned}
& I_{0}\left(\left\{n_{j}\right\}\right) \approx C \frac{\exp [F(0)]}{\sqrt{-F^{\prime \prime}(0)}}, \quad I_{1}\left(\left\{n_{j}\right\}\right) \approx C \frac{\exp [F(0)]}{\sqrt{-F^{\prime \prime}(0)+1}}, \\
& I\left(\left\{n_{j}\right\}\right) \equiv I_{0}\left(\left\{n_{j}\right\}\right)-I_{1}\left(\left\{n_{j}\right\}\right) \approx \frac{C}{2} \frac{\exp [F(0)]}{\left[-F^{\prime \prime}(0)\right]^{3 / 2}},
\end{aligned}
$$

where $C$ is just a constant and

$$
\begin{aligned}
& F(0)=\sum_{j} n_{j} \ln (2 j+1), \quad-F^{\prime \prime}(0)=\frac{1}{3} \sum_{j} n_{j} j(j+1)=\frac{\alpha A_{\mathrm{H}}}{4}, \\
& \alpha \equiv \frac{1}{6 \pi \gamma}\left(\frac{\sum_{j} f_{j} j(j+1)}{\sum_{j} f_{j} \sqrt{j(j+1)}}\right) .
\end{aligned}
$$

Here we have used the relation between the total number of punctures $p=\sum_{j} n_{j}$ and the horizon area: $A_{\mathrm{H}}=8 \pi \gamma p \sum_{j} f_{j} \sqrt{j(j+1)}$. From the results above, we have

$$
\frac{\delta \ln I}{\delta n_{j}} \approx \ln (2 j+1)-\left[\frac{2 j(j+1)}{\alpha A_{\mathrm{H}}}\right]
$$

so that, from equation (3.15), for the relevant configurations, the probability distribution for the fraction $f_{j}$ of the puncture sites with spin $j$ can be written as:

$$
f_{j} \equiv \frac{n_{j}}{\sum_{j} n_{j}} \approx(2 j+1) \exp \left[-\lambda \sqrt{j(j+1)}-\left(\frac{2 j(j+1)}{\alpha A_{\mathrm{H}}}\right)\right] .
$$

This provides an $\mathcal{O}\left(A_{\mathrm{H}}^{-1}\right)$ correction to the formula (3.11). Further, this probability distribution leads to the degeneracy of the horizon states given by:

$$
\mathcal{N}\left(\left\{n_{j}\right\}\right) \approx\left(\frac{4}{A_{\mathrm{H}}}\right)^{3 / 2} \exp \left[\left(\frac{\lambda}{2 \pi \gamma}\right) \frac{A_{\mathrm{H}}}{4}\right],
$$

whose logarithm yields the entropy formula for a large area black hole.

It is remarkable that this more careful calculation yields an entropy formula which has exactly the same form as that in equation (3.10) obtained earlier from the simplified calculation where all the punctures were assigned a common low value $r$ of spin. As has been pointed out in Section 3.1, the coefficient $-3 / 2$ of logarithmic correction is not sensitive to this common value $r$ of spin used in the calculations there. It is, therefore, no surprise that the more careful computation outlined above yields the same value for this coefficient. On the other hand, in the calculations of Section 3.1, the coefficient of the linear area term does depend on the common low value $r$ of the spin assigned to all the sites. In the more accurate calculation above also, we find that this coefficient indeed depends on how spins are distributed on the puncture sites. Fixing this coefficient by matching with the Bekenstein-Hawking area formula yields $\gamma=\frac{\lambda}{2 \pi}$. An important conclusion that follows from the improved calculations is that, from the 
distribution (3.11) or (3.16) for the maximized entropy, we now have a reliable estimate of the coefficient of the linear area term and consequently that of the Barbero-Immirzi parameter. Thus, for the value $\lambda \approx 1.72$ obtained by solving (3.12), we have $\gamma=\frac{\lambda}{2 \pi} \approx 0.27$. This value for $\gamma$ was first reported in [24], where the computations were done in the $U(1)$ framework in a way which is equivalent to the calculations above with $I_{1}$ put to zero, resulting in the coefficient of the logarithmic term as $-1 / 2$ in contrast to its correct value $-3 / 2$.

\section{$3.3 \quad S U(2)$ versus $U(1)$ formulations}

The $U(1)$ and $S U(2)$ Chern-Simons descriptions of horizon are some times viewed as counterpoints to each other and entropy calculations, particularly the logarithmic correction, in these two frameworks have been erroneously claimed to yield different results. These two points of view are in fact quite reconcilable. We have discussed this in the classical context earlier in Section 2: $U(1)$ formulation is merely a partially gauge fixed $S U(2)$ theory. As demonstrated there and emphasized earlier in [7], there are additional constraints in the $U(1)$ description. The Isolated Horizon boundary conditions which imply the $U(1)$ Chern-Simons equations (2.20) where the source $\Sigma_{\theta \phi}^{(1)}$ is one of the components of $S U(2)$ triplet of solder forms $\Sigma_{\theta \phi}^{(i)}=\gamma^{-1} e^{i j k} e_{\theta}^{i} e_{\phi}^{k}$, also imply the constraints $(2.21)$ for the solder forms $\Sigma_{\theta \phi}^{(2)}$ and $\Sigma_{\theta \phi}^{(3)}$ orthogonal to the $U(1)$ direction. These constraints essentially reflect the $S U(2)$ underpinnings of the classical $U(1)$ formulation. The quantum theory would have corresponding quantum version of these constraints. The horizon properties like associated entropy calculated from the quantum versions of these equivalent theories have to be exactly same. This is so as physics can not change by a gauge fixing in a gauge theory. To see that this is indeed so, care needs to be exercised by taking the quantum analogue of the classical conditions (2.21) into account in the calculations done in the $U(1)$ framework. These additional conditions play a crucial role in the computations and when properly implemented, exactly the same formula (3.10) for micro-canonical entropy of large area horizons follows in a straight forward manner. In the following, we shall briefly present an outline of this computation [7].

We wish to study the quantum formulation of the classical boundary $U(1)$ theory based on the action (2.9) and equations of motion (2.8) described in terms of the classical constraint: $-\frac{k}{2 \pi} F_{\theta \phi}=\Sigma_{\theta \phi}^{(1)}$, where $F_{\theta \phi}=\partial_{\theta} A_{\phi}-\partial_{\phi} A_{\theta}$ is the field strength of the boundary dynamical $U(1)$ gauge field $A_{\hat{a}}(\hat{a}=\theta, \phi)$. In the quantum boundary theory, the fields $\left(A_{\phi}, A_{\theta}\right)$ form a mutually conjugate canonical pair with commutation relation $\left[A_{\phi}\left(\sigma_{1}\right), A_{\theta}\left(\sigma_{2}\right)\right]=\frac{2 \pi i}{k} \delta^{(2)}\left(\sigma_{1}, \sigma_{2}\right)$. The solder form $\Sigma_{\theta \phi}^{(1)}$ is not a dynamical field in the boundary theory where it acts merely as an external source. On the other hand, the bulk quantum theory described by LQG is set up in terms of cylindrical functions made up of Wilson line operators of the bulk $S U(2)$ gauge fields as the configuration operators. The corresponding momentum operators are the fluxes with two-dimensional smearing $\int_{\mathbb{S}^{2}} d^{2} \sigma \Sigma_{\theta \phi}^{(i)}$ so that their Hamiltonian vector fields map cylindrical functions to cylindrical functions. The solder forms $\Sigma_{\theta \phi}^{(i)}$ have distributional support at the punctures where the spin networks impinge on the horizon. The hole micro-states $|\Psi\rangle$ of the quantum theory are composite states of the boundary theory and those of the bulk: $|\Psi\rangle \equiv$ $\mid$ boundary $\rangle \otimes \mid$ bulk $\rangle$ where the operators of the boundary theory act on the former and those of the bulk on the latter. Analogue of the classical IH boundary constraint of $U(1)$ formulation mentioned above has to be written in the quantum theory in terms of the flux operator (which is a dynamical operator of the bulk theory) instead of the solder form itself. Thus the physical states $|\Psi\rangle$ satisfy the quantum constraint: $-\frac{k}{2 \pi} \int_{\mathbb{S}^{2}} d^{2} \sigma F_{\theta \phi}|\Psi\rangle=\int_{\mathbb{S}^{2}} d^{2} \sigma \Sigma_{\theta \phi}^{(1)}|\Psi\rangle$, which relates the quantum flux through $\mathbb{S}^{2}$ of horizon in the boundary $U(1)$ gauge theory with the quantum flux of the bulk theory. Next, the $U(1)$ flux $\int_{\mathbb{S}^{2}} d^{2} \sigma F_{\theta \phi}$ in the boundary theory gets contributions, due 
to Stokes' theorem, from holonomies associated with the $p$ punctures as $\sum_{i=1}^{p} \oint_{C_{(i)}} d \sigma^{\hat{a}} A_{\hat{a}}$ where $\hat{a}=(\theta, \phi)$ and $\left\{C_{(i)}\right\}$ are $p$ non-intersecting loops in $\mathbb{S}^{2}$ such that $i$ th puncture is enclosed by a small loop $C_{(i)}$. This flux is equivalently given by the holonomy associated with a large closed loop $C$ enclosing all the punctures: $\oint_{C} d \sigma^{\hat{a}} A_{\hat{a}}$. Now, on $\mathbb{S}^{2}$, this large loop $C$ can be continuously shrunk on the other side to a point without crossing any of the punctures. This implies that the $U(1)$ flux $\int_{\mathbb{S}^{2}} d^{2} \sigma F_{\theta \phi}$ is zero on the physical states. Due to the quantum operator constraint mentioned above, this in turn leads to the fact that the bulk flux operator $\int_{\mathbb{S}^{2}} d^{2} \sigma \Sigma_{\theta \phi}^{(1)}$ annihilates the physical horizon states $|\Psi\rangle$ :

$$
-\frac{k}{2 \pi} \int_{\mathbb{S}^{2}} d^{2} \sigma F_{\theta \phi}|\Psi\rangle=\int_{\mathbb{S}^{2}} d^{2} \sigma \Sigma_{\theta \phi}^{(1)}|\Psi\rangle=0
$$

Similarly, the quantum analogue of the additional classical conditions (2.21) have to be written, instead of the solder forms $\Sigma_{\theta \phi}^{(2)}$ and $\Sigma_{\theta \phi}^{(3)}$, in terms of the dynamical operators of the bulk theory, namely the flux operators which act as derivations on the cylindrical functions (functionals of the holonomies). Consistent with these properties, the bulk fluxes acting on physical states $|\Psi\rangle$ of a spherically symmetric horizon, in addition to (3.17), satisfy the constraints:

$$
\int_{\mathbb{S}^{2}} d^{2} \sigma \Sigma_{\theta \phi}^{(2)}|\Psi\rangle=0, \quad \int_{\mathbb{S}^{2}} d^{2} \sigma \Sigma_{\theta \phi}^{(3)}|\Psi\rangle=0
$$

As stated earlier, the properties of the quantum operators $\Sigma_{\theta \phi}^{(i)}$ are completely determined by the bulk theory where these solder forms, acting on the spin networks, are distributional with support at the punctures where the network impinges on $\mathbb{S}^{2}$ of the horizon. In particular, the bulk flux operators, acting as derivations on spin network states of the bulk theory, satisfy a non-trivial commutation algebra: $\left[\int_{\mathbb{S}^{2}} d^{2} \sigma \Sigma_{\theta \phi}^{(i)}, \int_{\mathbb{S}^{2}} d^{2} \sigma \Sigma_{\theta \phi}^{(j)}\right]=i \epsilon^{i j k} \int_{\mathbb{S}^{2}} d^{2} \sigma \Sigma_{\theta \phi}^{(k)}$. The quantum constraints (3.18) are consistent with this property. These additional conditions only reflect the underlying $S U(2)$ invariance of the gauge fixed quantum boundary theory described in terms of $U(1)$ gauge fields and are merely the quantum analogues of the constraints (2.21) of the classical $U(1)$ theory written in terms of the dynamical operators of the bulk quantum theory.

Conversely, it is straightforward to check that the additional conditions (3.18) of the $U(1)$ formulation indeed follow directly from the partial gauge fixing of the quantum $S U(2)$ ChernSimons boundary theory. This $S U(2)$ formulation is described by a quantum constraint in terms of the exponentiated form of the flux operators acting on the physical states $|\Psi\rangle$ as: $\mathcal{P} \exp \left(\int_{\mathbb{S}^{2}} d^{2} \sigma \Sigma_{\theta \phi}^{(i)} T^{(i)}\right)|\Psi\rangle=\mathcal{P} \exp \left(-\frac{k}{2 \pi} \int_{\mathbb{S}^{2}} d^{2} \sigma F_{\theta \phi}\right)|\Psi\rangle$, where $T^{(i)}$ is a basis of $S U(2)$ algebra and $F_{\theta \phi} \equiv F_{\theta \phi}^{(i)} T^{(i)}$ is the field strength of the boundary $S U(2)$ gauge field $A_{\hat{a}} \equiv A_{\hat{a}}^{(i)} T^{(i)}$. Here the symbol $\mathcal{P}$ represents surface (path) ordering in a specific way consistent with the nonAbelian Stokes' theorem [25, 26]. This constraint relates the flux functional of the bulk theory to that of the boundary gauge theory. The $S U(2)$ gauge transformations act on these flux functionals as conjugations. In the boundary Chern-Simons theory, $S U(2)$ quantum gauge fields $\left(A_{\phi}^{(i)}, A_{\theta}^{(i)}\right)$ are mutually conjugate with their commutation relations as: $\left[A_{\phi}^{(i)}\left(\sigma_{1}\right), A_{\theta}^{(j)}\left(\sigma_{2}\right)\right]=$ $\frac{2 \pi i}{k} \delta^{i j} \delta^{(2)}\left(\sigma_{1}, \sigma_{2}\right)$. Consequently, the boundary gauge fluxes $-\frac{k}{2 \pi} \int_{\mathbb{S}^{2}} d^{2} \sigma F_{\theta \phi}^{(i)}$ do not commute; in fact, it is easy to check that these obey $S U(2)$ Lie algebra commutation rules:

$$
\left[\frac{k}{2 \pi} \int_{\mathbb{S}^{2}} d^{2} \sigma F_{\theta \phi}^{(i)}, \frac{k}{2 \pi} \int_{\mathbb{S}^{2}} d^{2} \sigma F_{\theta \phi}^{(j)}\right]=-i \epsilon^{i j k} \frac{k}{2 \pi} \int_{\mathbb{S}^{2}} d^{2} \sigma F_{\theta \phi}^{(k)}
$$

In a similar manner,as pointed out earlier, the bulk flux operators $\int_{\mathbb{S}^{2}} d^{2} \sigma \Sigma_{\theta \phi}^{(i)}$ also satisfy $S U(2)$ Lie algebra commutation rules. Therefore, this introduces ordering ambiguities in the definition 
of the surface ordered boundary and bulk flux functionals used here. However, these ambiguities can be fixed by using the Duflo map which provides a quantization map for functions on Lie algebras [27, 26]. Now, a non-Abelian generalization [25] of the Stokes' theorem allows us to replace the surface ordered boundary gauge flux functional depending on the $S U(2)$ field strength by a related path ordered holonomy functional of the corresponding boundary $S U(2)$ gauge connection: $\mathcal{P} \exp \left(-\frac{k}{2 \pi} \int_{\mathbb{S}^{2}} d^{2} \sigma F_{\theta \phi}\right)=\mathcal{P} \exp \left(-\frac{k}{2 \pi} \oint_{C} d \sigma^{\hat{a}} A_{\hat{a}}\right)$, where $C$ is a contour enclosing all the punctures. Since this contour $C$ can be contracted to a point on $\mathbb{S}^{2}$, this holonomy functional is simply equal to 1 on the physical states so that the quantum fluxes of the bulk and boundary theories acting on the physical states $|\Psi\rangle$ satisfy the constraint:

$$
\mathcal{P} \exp \left(\int_{\mathbb{S}^{2}} d^{2} \sigma \Sigma_{\theta \phi}^{(i)} T^{(i)}\right)|\Psi\rangle=\mathcal{P} \exp \left(-\frac{k}{2 \pi} \int_{\mathbb{S}^{2}} d^{2} \sigma F_{\theta \phi}^{(i)} T^{(i)}\right)|\Psi\rangle=|\Psi\rangle .
$$

The degeneracy of the black hole quantum states may be calculated in the boundary $S U(2)$ Chern-Simons theory by counting those states where the functional of gauge flux operator evaluated over the punctured $\mathbb{S}^{2}$ of the horizon has eigenvalue 1 . The result is obtained by counting of the number of ways singlets can be constructed by composing the spins $j_{i}$ on the punctures in the $S U(2)$ Chern-Simons theory. This is exactly how the computations outlined in Sections 3.1 and 3.2 have been performed. Equivalently, this degeneracy may also be calculated by counting the bulk spin network states on which the bulk flux functional has eigenvalue 1 . Note that the punctures carrying the spins $j_{i}$ on the $\mathbb{S}^{2}$ of the horizon are common to the boundary and bulk states which are connected by the functional flux constraint. This ensures that the counting done in these two ways, in the boundary theory and in the bulk theory, yield the same results. Next, the boundary $S U(2)$ quantum Chern-Simons theory can be partially gauge fixed to a gauge theory based on the maximal torus group $T=U(1)$ of $S U(2)$ through appropriate gauge conditions on the boundary gauge fields such that the gauge fluxes in the two internal directions orthogonal to this $U(1)$ subgroup are zero: $\int_{\mathbb{S}^{2}} d^{2} \sigma F_{\theta \phi}^{(2)}=0$ and $\int_{\mathbb{S}^{2}} d^{2} \sigma F_{\theta \phi}^{(3)}=0$. This Abelian reduction converts the flux constraint of the $S U(2)$ formulation to that of the $U(1)$ formulation:

$$
\exp \left(\int_{\mathbb{S}^{2}} d^{2} \sigma \Sigma_{\theta \phi}^{(1)}\right)|\Psi\rangle=\exp \left(-\frac{k}{2 \pi} \int_{\mathbb{S}^{2}} d^{2} \sigma F_{\theta \phi}\right)|\Psi\rangle=|\Psi\rangle
$$

where now $F_{\theta \phi}$ here is the field strength of the boundary $U(1)$ gauge field, along with the additional quantum conditions for the bulk fluxes (3.18): $\int_{\mathbb{S}^{2}} d^{2} \sigma \Sigma_{\theta \phi}^{(2)}|\Psi\rangle=0$ and $\int_{\mathbb{S}^{2}} d^{2} \sigma \Sigma_{\theta \phi}^{(3)}|\Psi\rangle=0$.

Now the horizon entropy in the $U(1)$ formulation is obtained by counting, for a fixed large area, the number of ways the spins $j_{1}, j_{2}, \ldots, j_{p}$ can be placed on the $p$ punctures so that their $U(1)$ projection eigenvalues, the $m$-quantum numbers of the diagonal flux operator $\int_{\mathbb{S}^{2}} d^{2} \sigma \Sigma_{\theta \phi}^{(1)}$, add up to zero, $m_{\text {tot }} \equiv \sum_{l=1}^{p} m_{l}=0$, to ensure that the physical states $|\Psi\rangle$ satisfy the $U(1)$ constraint (3.17). Notice that these $m_{\text {tot }}=0$ configurations include all such states from the irreducible representations with $\operatorname{spin} j_{\text {tot }}=0,1,2,3, \ldots$ in the tensor product $\otimes_{l=1}^{p}\left(j_{l}\right)$. Total number of these configurations is counted exactly by the first term of the degeneracy formula (3.8) in the large $k$ limit $(k \gg 1)$ where the periodic Kronecker delta $\bar{\delta}_{m, n}$ becomes ordinary delta $\delta_{m, n}$. Now, if we ignore the additional constraints (3.18) of the quantum theory, from the first term of (3.8), we shall get the entropy with the leading area term and a sub-leading $\ln A_{\mathrm{H}}$ correction with coefficient $-1 / 2$ as has been done in several places $[15,23,24]$. But this is clearly an over counting of the horizon states as the correct counting would require to exclude those states with $\sum_{l=1}^{p} m_{l}=0$ which do not respect these additional constraints. To reiterate, the additional quantum constraints (3.18) require that only physical states to be counted for a non-rotating horizon 
are those which belong to the kernel of the ladder generators $J^{( \pm)} \equiv \int_{\mathbb{S}^{2}} d^{2} \sigma \frac{1}{2}\left[\Sigma_{\theta \phi}^{(2)} \pm i \Sigma_{\theta \phi}^{(3)}\right]$ of the total spin algebra, besides being in the kernel of the diagonal generator $J^{(1)} \equiv \int_{\mathbb{S}^{2}} d^{2} \sigma \Sigma_{\theta \phi}^{(1)}$ with eigenvalues $m_{\text {tot }}=0$. Now, acting on some of the vectors in the kernel of the diagonal generator, the ladder generators $J^{( \pm)}$map them to states with $m$-quantum number, $m_{\text {tot }} \equiv \sum_{l=1}^{p} m_{l}= \pm 1$. These are the states with $m_{\text {tot }}=0$ from total spin $j_{\text {tot }}=1,2,3, \ldots$ states in the tensor product representation $\otimes_{l=1}^{p}\left(j_{l}\right)$. Thus the quantum constraints (3.18) require that such states should not be included in the count. The second and third terms in the formula (3.8) (in the large $k$ limit) precisely count these extra states. Since the $m_{\text {tot }}=+1$ and $m_{\text {tot }}=-1$ components occur in a non-zero integer spin state in equal numbers, we have the normalization coefficient $1 / 2$ in front of each of these two terms in (3.8). Thus, even in the gauge fixed formulation described in terms of quantum $U(1)$ Chern-Simons theory with correctly identified additional quantum constraints (3.18), a careful counting leads to the same formula (3.8) as in the $S U(2)$ framework; the asymptotic entropy formula (3.10) with the coefficient $-3 / 2$ for the leading $\log$ (area) correction holds in both the formulations. Additionally, the value of Barbero-Immirzi parameter $\gamma$ fixed through matching of the area term with Bekenstein-Hawking law is also the same. These results are not surprising but merely a reflection of the fact that gauge invariance requires that physical quantities do not change by gauge fixing.

In fact, like in any other gauge theory, we could further fix the gauge in the $U(1)$ formulation so that whole of the gauge invariance of the boundary theory is now fixed. Gauge invariance would imply that counting of the relevant states in this formulation should again yield the same result for black hole entropy as that in the formulation with full $S U(2)$ gauge invariance.

\section{Black hole entropy from other perspectives}

Black hole entropy has also been calculated in quantum frameworks other than that provided by LQG. These lead to several derivations of the asymptotic entropy formula (3.10) for a variety of black holes. This includes those for many black holes in the String Theory. This entropy formula appears to hold even for black holes of theories in dimensions other than four. We shall briefly survey a few of these cases here.

\subsection{Entropy from Cardy formula}

Immediately after the discovery of $-(3 / 2) \ln A_{\mathrm{H}}$ correction to the Bekenstein-Hawking area law obtained from the $S U(2)$ Chern-Simons theory of horizon in LQG [14], Carlip demonstrated that this is in fact a generic feature of any conformal field theory independent of its detail structure [28]. This important result was derived by a careful calculation of the logarithmic correction to the Cardy formula. The number density of states $\rho(\Delta)$ with the eigenvalue $\Delta$ of the generator $L_{0}$ of Virasoro algebra in a conformal field theory with central charge $c$, for large $\Delta$, was shown to be:

$$
\rho(\Delta) \sim\left(\frac{c}{96 \Delta^{3}}\right)^{1 / 4} \exp \left(2 \pi \sqrt{\frac{c \Delta}{6}}\right) .
$$

The exponential term is the Cardy formula [29] and the fore-factor provides logarithmic correction to it. Derivation of this result does not require any detail knowledge of the partition function of conformal field theory; all that goes in to the calculations is the generic modular transformation properties of the torus partition function.

The Carlip formula (4.1) is of particular interest as there are strong suggestions that conformal field theories do indeed provide a universal description of low energy properties of black holes [30] 
which is relevant even in the framework of String Theory. For the case where black hole horizon properties are described by a single conformal theory, the argument of the exponential in (4.1) can be identified with the Bekenstein-Hawking entropy, $S_{\mathrm{BH}}=2 \pi \sqrt{c \Delta / 6}$. This is the case with many black holes in the String Theory. Thus, for such a model, the Carlip formula readily yields horizon entropy as:

$$
S_{\mathrm{H}}=\ln \rho(\Delta)=S_{\mathrm{BH}}-\frac{3}{2} \ln S_{\mathrm{BH}}+\ln c+\cdots
$$

with its first two terms same as in the LQG entropy formula (3.10). Carlip has applied this result to analyse several cases which include the BTZ black hole in $2+1$ dimensions and string theoretic counting of D-brane states for BPS black holes [31]. With this, presence of logarithmic correction with the definite coefficient $-3 / 2$ for many black holes in three, four and higher dimensions has been established. Carlip has made an eloquent case for the universal nature of this logarithmic correction.

In another derivation of black hole entropy from the conformal field theory perspective, instead of the corrected Cardy formula, Rademacher's exact convergent expansion for the Fourier coefficients of a modular form of a given weight has been used in [32]. This analysis also shows that, for large holes, the leading logarithmic correction to the entropy has the universal coefficient $-3 / 2$, again in conformity with the LQG formula (3.10).

\subsection{Entropy of BTZ black hole in the Euclidean path integral approach}

There are alternative methods, besides those described above, to study the entropy of BTZ hole. In fact, it is possible to derive an exact expression for the partition function of Euclidean BTZ black hole in the path integral approach [33]. Entropy for a large area Lorentzian BTZ hole is extracted from this after a proper analytic continuation.

We start by writing three-dimensional Euclidean gravity with a negative cosmological constant in the first order formulation (with triads $e$ and spin connection $\omega$ ) in terms of two $S U(2)$ Chern-Simons theories [34]:

$$
I_{\text {grav }}=k I_{\mathrm{CS}}[A]-k I_{\mathrm{CS}}[\bar{A}] \text {, }
$$

where $I_{\mathrm{CS}}$ represents the Chern-Simons action for complex gauge fields $A=\left(i \ell^{-1} e^{i}+\omega^{i}\right) T^{i}$ and $\bar{A}=\left(i \ell^{-1} e^{i}-\omega^{i}\right) T^{i}$ with $T^{i} \equiv i \sigma^{i} / 2$ as the generators of the Lie algebra of $S U(2)$ and coupling $k=\ell /(4 G)$ for negative cosmological constant $\Lambda=-1 / \ell^{2}$. The gauge group of this theory is $S L(2, \mathbb{C})$. Corresponding gauge group for Minkowski gravity with negative cosmological constant is $S O(2, \mathbb{R}) \otimes S O(2, \mathbb{R})$ with coupling $k=-\ell /(4 G)$. The Lorentzian results are obtained from Euclidean theory after completing the computations of various quantities of interest by an analytic continuation $G \rightarrow-G$.

Taking time and angular momentum to be pure imaginary as $t=i \tau_{\mathrm{E}}$ and $J=-i J_{\mathrm{E}}$ and consequently the inner horizon radius $r_{-}=-i\left|r_{\mathrm{E}-}\right|$, we obtain the Euclidean continuation of BTZ black hole which has the topology of a solid torus [35]. On a solid torus, of the two nontrivial cycles of the boundary 2-torus, one becomes contractible while other is non-contractible. We are interested in the path integral for the Chern-Simons theory (4.3) on a solid torus with a boundary modular parameter $\tau=\tau_{1}+i \tau_{2}$. This is evaluated by keeping the trace of gauge field holonomy along the non-contractible cycle on the boundary 2-torus fixed. This holonomy is a function of the outer (event) and inner horizon radii $r_{+}$and $r_{-}$which are given in terms of the mass parameter $M$ and angular momentum $J$ of the hole and hence, this keeps these quantities fixed. The quantum fluctuations are introduced through Wilson lines with spin $n / 2$ along the non-contractible cycle inside the solid torus. These create defect angles (characterized by the spin $n / 2$ ) at the horizon which are not kept fixed and all possible values are included 
in the path integral by summing over various values of the spin $n / 2$ (with $n \leq k$ ). The states corresponding to these closed Wilson lines are given by [36]:

$$
\psi_{n}(u, \tau)=\exp \left(\frac{\pi k u^{2}}{4 \tau_{2}}\right) \chi_{n}(u, \tau), \quad n=0,1,2, \ldots, k,
$$

where $u=-\tau-i\left(r_{+}+i\left|r_{-}\right|\right) / \ell$ characterizes the boundary value of the gauge connection and $\chi_{n}$ are the Weyl-Kac characters for affine $S U(2)$ which are given in terms of the Theta functions as:

$$
\chi_{n}(u, \tau)=\frac{\Theta_{n+1}^{(k+2)}(u, \tau, 0)-\Theta_{-n-1}^{(k+2)}(u, \tau, 0)}{\Theta_{1}^{(2)}(u, \tau, 0)-\Theta_{-1}^{(2)}(u, \tau, 0)}
$$

with

$$
\Theta_{n}^{(k)}(u, \tau, z)=\exp (-2 \pi i k z) \sum_{s \in \mathbb{Z}} \exp \left\{2 \pi i k\left[\left(s+\frac{n}{2 k}\right)^{2} \tau+\left(s+\frac{n}{2 k}\right) u\right]\right\} .
$$

Finally, the Euclidean black hole partition function is given by [33]:

$$
Z_{\mathrm{E}}=\int d \mu(\tau, \bar{\tau})\left|\sum_{n=0}^{k}\left(\psi_{n}(0, \tau)\right)^{*} \psi_{n}(u, \tau)\right|^{2} .
$$

Here the integrand is invariant under the 2-torus modular transformations $\mathcal{S}: \tau \rightarrow-1 / \tau$, $u \rightarrow u / \tau$ and $\mathcal{T}: \tau \rightarrow \tau+1$. The integration is done with the modular invariant measure: $d \mu(\tau, \bar{\tau})=(d \tau d \bar{\tau}) / \tau_{2}^{2}$ where $\tau_{2}=\operatorname{Im} \tau$.

The result (4.4) is an exact expression for the partition function of a Euclidean black hole. We may now evaluate it for large horizon radius $r_{+}\left(r_{+}>\ell>4 G\right)$ by the saddle point method. The saddle point of the integrand is at a large value of $\operatorname{Im} \tau$ given by $\tau_{2}=r_{+} / \ell$ for $\left|r_{-}\right| \ll r_{+}$. The computation is done for a positive coupling constant $k=\ell /(4 G)$ and in the end, we go over to the Lorentzian black hole through the analytic continuation $G \rightarrow-G$. After this analytic continuation, it can be shown that $\operatorname{spin} n=0$ term dominates in the sum in (4.4). Finally, for large horizon length $r_{+}\left(r_{+} \gg \ell\right.$ with $\left.r_{-} \ll r_{+}\right)$, this procedure leads to the Lorentzian formula [33]:

$$
Z_{\mathrm{L}} \sim \frac{\ell^{2}}{r_{+}^{2}} \sqrt{\frac{8 r_{+} G}{\pi \ell^{2}}} \exp \left(\frac{2 \pi r_{+}}{4 G}\right),
$$

whose logarithm yields an asymptotic formula for the entropy of Lorentzian BTZ hole:

$$
S_{\mathrm{BTZ}}=\frac{2 \pi r_{+}}{4 G}-\frac{3}{2} \ln \left(\frac{2 \pi r_{+}}{4 G}\right)+\ln k+\cdots
$$

with $k=\ell /(4 G)$. The leading Bekenstein-Hawking term was already obtained earlier from other Euclidean calculations [37]. The new computation reviewed here provided the sub-leading logarithmic correction with coefficient $-3 / 2$ in agreement with the results obtained for the BTZ hole from the corrected asymptotic Cardy formula (4.1) and also with the LQG result (3.10) for the holes in four dimensional theory.

As pointed out above, the asymptotic formula (4.5) holds for large $r_{+}\left(r_{+} \gg \ell \gg 4 G\right)$. However, for smaller $r_{+}$, different results hold [38]: (i) For $r_{+} \sim \ell$, where the saddle point of the integrand in (4.4) occurs at $\tau_{2} \sim 1$, the entropy is given by $S_{\mathrm{BTZ}}=\frac{2 \pi r_{+}}{4 G}-\ln \left(\frac{2 \pi r_{+}}{4 G}\right)+\cdots$ This is also the entropy associated with the cosmological horizon of three dimensional Lorentzian de Sitter space. (ii) On the other hand, for $r_{+} \ll \ell$, we have $S_{\mathrm{BTZ}}=\frac{2 \pi \ell^{2}}{4 r_{+} G}+\frac{3}{2} \ln \left(\frac{r_{+}}{\ell}\right)+\cdots$ which represents the entropy of AdS gas. 


\subsection{Entropy of a highly excited string}

It is more than two decades now since 't Hooft suggested a complementarity between black holes and strings [39]: it may be possible to provide a black hole interpretation of strings and conversely, black holes may have a string representation. Susskind's idea [40] that the microstates of a Schwarzschild black hole could be described by the states of a highly excited string at the Hagedron temperature may be viewed as a reflection of this complementarity with more evidence provided in [41] and others. This correspondence principle for the two spectra can be understood as follows: As the string coupling $g_{\text {str }} \equiv\left(\ell_{\mathrm{P}} / \ell_{\mathrm{S}}\right)^{(d-2)}$ in $d$ dimensions $\left(\ell_{\mathrm{P}}\right.$ is the Planck length and $\ell_{\mathrm{S}}$ is the string scale) increases, the Compton wave-length of a high mass and low angular momentum state of the string shrinks to a size smaller than its Schwarzschild radius and it becomes a black hole. Conversely, as the coupling is reduced, the hole becomes smaller and at some stage it is smaller than the string size. The metric near horizon loses it meaning and instead of the hole, we have an object which is better described as a string state. At some in between stage, when string and hole sizes are equal, either description is possible implying a one-to-one correspondence between the two spectra [40]. Using the fact that near horizon geometry of a Schwarzschild black hole is a Rindler space, Susskind has suggested that square root of the oscillator number $\sqrt{N}$ of the highly excited string should be identified with the Rindler energy $E_{\mathrm{R}}$ instead of the ADM mass of the hole. Rindler energy and ADM mass are related by a huge red shift between the stretched horizon and asymptotic infinity. For the Schwarzschild black hole in $d$ dimensions $(d \geq 4)$, Rindler energy is linearly related to the horizon area: $A_{\mathrm{H}}=8 \pi G E_{\mathrm{R}}$ and hence the Bekenstein-Hawking entropy is $S_{\mathrm{BH}}=2 \pi E_{\mathrm{R}}$.

To push this correspondence further, we may calculate the density of states $\rho(N)$ with high oscillator number $N$ in a string theory. For an open bosonic string moving in $d$ dimensions, the partition function as a function of a complex parameter $\tau$ is given by [42]:

$$
Z(\tau)=\left(\frac{1}{-i \tau}\right)^{(d-2) / 2} e^{-2 \pi i \tau a} \operatorname{tr} \exp [2 \pi i \tau \mathcal{N}]
$$

where $c=24 a=(d-2)$ is the central charge and $\mathcal{N}$ is the occupation number operator with its eigenvalues represented by $N$. It is given in terms of the oscillator number operators $\mathcal{N}_{m}$ as $\mathcal{N}=\sum_{m=1}^{\infty} m \mathcal{N}_{m}$. Here $\mathcal{N}_{m}=\sum_{i=1}^{d-2} a_{m}^{i \dagger} a_{m}^{i}$ with $a_{m}^{i \dagger}$ and $a_{m}^{i}$, with $i=1,2, \ldots,(d-2)$, as the standard oscillator creation and destruction operators associated with the transverse $(d-2)$ dimensions in the light-cone gauge. The eigenvalues of the oscillator number operator $\mathcal{N}_{m}$ are $0,1,2,3, \ldots$ The expression for partition function above has been derived with care by taking in to account the contribution of zero modes which has resulted in the fore-factor $(-i \tau)^{-(d-2) / 2}$. The trace over the oscillator states is done using number theory techniques involving the number of partitions of $N$ in terms of positive integers and modular transformation properties of the partition function.

We may alternatively write the partition function in terms of the level density $\rho(N)$ for the

states with eigenvalue $N$ of the number operator $\mathcal{N}$ as: $Z(\tau)=\sum_{N=0}^{\infty} \rho(N) e^{2 \pi i(N-a) \tau}$ which can be inverted to write a formula for $\rho(N)$ in terms of the partition function. After a modular transformation $\tau \rightarrow-1 / \tau$, this is then evaluated by the saddle point method for large $N$ to yield the result [42]:

$$
\rho(N) \sim C \frac{a \exp (4 \pi \sqrt{a N})}{(a N)^{3 / 4}},
$$

where $C$ is an $N$ independent constant. This equation is only a special case of the formula (4.1) obtained by Carlip for a general conformal field theory. 
Now, as suggested by Susskind, we identify the Rindler energy as $E_{\mathrm{R}}=2 \sqrt{a N}$. This leads to an entropy formula for this highly excited string moving in $d$ dimensions, given by the logarithm of the level density $\rho(N)$, as:

$$
S_{\mathrm{str}}=2 \pi E_{\mathrm{R}}-\frac{3}{2} \ln E_{\mathrm{R}}+\ln a+\cdots .
$$

Though the asymptotic formula for level density above was calculated for an open bosonic string, it is valid in general for any string theory. Again the logarithmic correction with coefficient $-3 / 2$ matches with the LQG entropy formula (3.10). This result may be interpreted as an additional evidence for the excited string $\leftrightarrow$ black hole correspondence.

Similar to the string calculation above, a more general study of the asymptotic density of states for open $p$-branes has been done by Kalyana Rama in [43]. Careful inclusion of the contributions of zero modes here leads to a logarithmic correction to the entropy with coefficient $-(p+2) /(2 p)$ which agrees with string result $-3 / 2$ for $p=1$.

We have surveyed some of the alternative derivations of the asymptotic entropy formula (3.10). There are still several others [44, 45], particularly with the same leading logarithmic correction as in (3.10), that have appeared over the years. Of these the most recent one is by Davidson where a discrete holographic shell model is proposed for a spherical black hole [45]. Instead of putting them on punctures or small Planck scale patches on the horizon, the degrees of freedom are distributed holographically in the entire black hole interior in concentric spherical shells of light sheet unit intervals. The number of distinguishable configurations is given by the Catalan series, which readily leads to an asymptotic entropy formula with a logarithmic correction with coefficient $-3 / 2$. As pointed out in [45], it is of interest to note that Catalan numbers are directly related to a standard stack data structure for storage of information in computer science. In this context, we may also recall equation (3.9) from Section 3.1, which represents the fact that $S U(2)$ singlets in the composite representation made of spin $1 / 2$ assignments on all the $p$ punctures on $\mathbb{S}^{2}$ of horizon are precisely counted by the Catalan number $C_{n}=\frac{(2 n) !}{(n+1) ! n !}$ for $p=2 n$.

\subsection{Universality of the logarithmic correction}

As we have listed above, the leading logarithmic correction, $-3 / 2 \ln A_{\mathrm{H}}$, to the BekensteinHawking area law found first in LQG appears to obtain from a variety of other perspectives, conceptually distinct from the LQG framework. The same logarithmic correction has also been derived for black holes in theories in dimensions other than four. For example, BTZ black holes of three dimensional gravity do exhibit this property. It is remarkable, though apparently mysterious, that such diverse approaches should lead to the same result. For all those models where ultimately the black hole properties are represented by conformal field theories, Carlip's work has demonstrated that such a correction is generic. This holds for many black holes in the string theory. Besides this logarithmic term, conformal field theory result (4.2) also has a $\ln c$ correction depending on the central charge. Power law dependence of $c$ on the area, would change the coefficient $-3 / 2$ of the $\ln A_{\mathrm{H}}$ term. Thus for those theories where central charge is independent of area, the LQG asymptotic formula (3.10) holds. All these facts suggest a strong case for the universal character of the logarithmic area correction.

\section{Recent developments}

In last few years, there has been a resurgent interest in the $S U(2)$ gauge theoretic description of Isolated Horizons within LQG framework [8, 46, 47, 48, 49]. The $S U(2)$ Chern-Simons theory 
as a description of the horizon has been re-emphasized in [8] which has been followed by further work in $[46,47,48]$ and others. This has been presented as an alternative to the $U(1)$ ChernSimons formulation. However, as we have seen above these two formulations provide equivalent descriptions with exactly same consequences. A direct representation of the black hole states in terms of $S U(2)$ intertwining operators has also been explored in [49]. The Hilbert space of these operators is the same as those of the $S U(2)$ Chern-Simons states in the limit of large coupling $k$ $(k \gg 1)$.

Some of these recent papers $[8,46,47]$ have recalculated horizon entropy in the $S U(2)$ ChernSimons framework and reconfirmed the form of asymptotic entropy formula (3.10), particularly with the -(3/2) $\ln A_{\mathrm{H}}$ correction. For example, calculations in first two papers in [46] start with the standard integral representation in terms of $S U(2)$ characters for the degeneracy of $S U(2)$ singlet states in the composite representation $\otimes_{l=1}^{p}\left(j_{l}\right)$. This is just an integral representation of the degeneracy formula (3.4) (or equivalently (3.7) or (3.8)) in the limit of large $k$ $\left(=\frac{A_{\mathrm{H}}}{4 \gamma} \gg 1\right)$ where these $S U(2)_{k}$ formulae can be approximated by those for ordinary $S U(2)$. The various spin values $j$ are distributed over the punctures with varying occupancy numbers $n_{j}$. The degeneracy of black hole states is to be obtained by restricting to configurations

which yield area values in the interval $\left[A_{\mathrm{H}}-\epsilon, A_{\mathrm{H}}+\epsilon\right]$ with $A_{\mathrm{H}}=8 \pi \gamma \sum_{l=1}^{p} \sqrt{j_{l}\left(j_{l}+1\right)}$ for a reasonable choice of $\epsilon$. To solve various relevant combinatorial constraints, powerful number theory techniques developed earlier [50] have been used to obtain generating functions from which the degeneracy of black hole states has been extracted through the Laplace transform method. This then reproduces, for large area, the logarithmic correction with the definite coefficient $-3 / 2$ as found earlier in [14] and reviewed here in Section 3.1. Besides this, the formula (3.12), earlier found in [23, 24], is also obtained. Consequently the improved value of the Barbero-Immirzi parameter mentioned in Section 3.2 and first found in [24], is recovered. These calculations have been done using ordinary $S U(2)$ counting rules, instead of the full $S U(2)_{k}$ formulae. These are adequate to yield the leading linear area and logarithmic terms of the asymptotic formula (3.10). An important feature of these computations is that, when extended to include effects depending on the smaller values of $k$, these may also provide a method to study the properties of small black holes where such effects would be important.

Lastly, an effective bulk gravity action of the form $f(R)$ that reproduces the asymptotic LQG black hole entropy with its logarithmic correction through the Wald procedure has also been derived recently in [51].

\section{Concluding remarks}

We have here surveyed how the Chern-Simons theoretical description of horizon degrees of freedom emerges in the LQG. This leads to two formulations of the theory with $S U(2)$ and $U(1)$ gauge invariances. It has been demonstrated that these provide equivalent descriptions; the latter being only a gauge fixed version of the former. A framework developed more than a decade ago, that relates the $S U(2)$ Chern-Simons theory to gauged $S U(2)_{k}$ Wess-Zumino conformal field theory, to compute the horizon entropy has been presented. We have also discussed the calculations in the equivalent quantum $U(1)$ formulation. When carefully identified additional quantum constraints (3.18) are properly implemented, this formulation also yields the same results as the earlier $S U(2)$ theory. This is in conformity with fact that gauge fixing does not change the physical properties. Besides the Bekenstein-Hawking area term, the micro-canonical entropy possesses a leading correction as logarithm of the horizon area with coefficient $-3 / 2$ for large horizons. This is followed by further corrections which are a constant and terms containing inverse powers of area. These corrections have their origin in the non-perturbative quantum 
fluctuations of geometry in contrast to those that come from quantum matter fluctuations. The logarithmic correction for black holes in four dimensional gravity has since been obtained in other frameworks too. These results appear to be valid for black holes in other than four dimensions as well. We have outlined some of these developments here. The logarithmic correction to the Bekenstein-Hawking entropy may possibly have a universal character.

Derivation of leading terms of the asymptotic entropy formula (3.10) from the Chern-Simons theory of horizon does not require the full force of $S U(2)_{k}$ conformal field theory; these terms emerge by using the counting rules of ordinary $S U(2)$ which corresponds to the large $k$ limit $(k \gg 1)$ of conformal theory. However, the terms beyond logarithmic correction are sensitive to the smaller values of $k$. Further, the general framework explored through the boundary conformal theory $[13,14]$ discussed here needs to be exploited to unravel the properties of small black holes. Effect of the smaller values of $k\left(=\frac{A_{\mathrm{H}}}{4 \gamma}\right)$ would be pronounced for such holes and hence $S U(2)_{k}$ conformal field theory will play important role here.

In the survey here, Isolated Horizons have been studied in the fixed area ensemble where micro-canonical entropy emerges from the micro-states associated with quantum geometry degrees of freedom of horizon. We have not discussed the entropy of radiant black holes in thermal equilibrium with their radiation bath. These are described by a canonical ensemble with fixed area and energy. The canonical entropy of such a hole results from both the quantum geometry fluctuations of horizon as well as the thermal fluctuations. While the counting of quantum geometry micro-states leads to a negative correction to the BekensteinHawking law, the thermal fluctuations due to exchange of heat between the hole and its surroundings, which increase the uncertainty of the horizon area, would lead to a positive correction. We shall now close our discussion with a few remarks about some recent results about canonical entropy of these holes. A general analysis to study radiant spherical holes has been set up in $[52,53,54]$. Canonical entropy of such holes has been calculated [54] to be given by the micro-canonical entropy $S_{\text {micro }}\left(A_{\mathrm{H}}\right)$ emerging from quantum geometry degrees of freedom with an extra correction due to the thermal fluctuations as: $-\frac{1}{2} \ln \Delta\left(A_{\mathrm{H}}\right)$ where $\Delta\left(A_{\mathrm{H}}\right)$ is given in terms of the mass function $M\left(A_{\mathrm{H}}\right)$ and micro-canonical entropy by $M^{\prime}\left(A_{\mathrm{H}}\right) S_{\text {micro }}^{\prime 2}\left(A_{\mathrm{H}}\right) \Delta\left(A_{\mathrm{H}}\right)=a\left[M^{\prime \prime}\left(A_{\mathrm{H}}\right) S_{\text {micro }}^{\prime}\left(A_{\mathrm{H}}\right)-M^{\prime}\left(A_{\mathrm{H}}\right) S_{\text {micro }}^{\prime \prime}\left(A_{\mathrm{H}}\right)\right]$. Here prime denotes derivative with respect to the argument and $a$ is a positive constant. Also the heat capacity is $C=\left[S_{\text {micro }}^{\prime}\left(A_{\mathrm{H}}\right)\right]^{2} / \Delta\left(A_{\mathrm{H}}\right)$. This provides a universal criterion for thermal stability of holes [54]: positivity of $\Delta\left(A_{\mathrm{H}}\right)$ or equivalently the integrated condition $M\left(A_{\mathrm{H}}\right)>S_{\text {micro }}\left(A_{\mathrm{H}}\right)$ ensures thermal stability. Clearly for the Schwarzschild black hole where $M\left(A_{\mathrm{H}}\right) \sim \sqrt{A_{\mathrm{H}}}$, we have $\Delta<0$ reflecting the usual thermal instability. On the other hand, for AdS Schwarzschild hole, the mass-area relation is $M\left(A_{\mathrm{H}}\right)=\frac{1}{2} \sqrt{\frac{A_{\mathrm{H}}}{2 \pi}}\left(1+\frac{A_{\mathrm{H}}}{4 \pi \ell^{2}}\right)$ with $-1 / \ell^{2}$ as the cosmological constant. Here, for small areas, $A_{\mathrm{H}}<4 \pi \ell^{2}$, where $M\left(A_{\mathrm{H}}\right)$ has the ordinary Schwarzschild like behaviour $M\left(A_{\mathrm{H}}\right) \sim \sqrt{A}_{\mathrm{H}}$, we have thermal instability. For large areas, $A_{\mathrm{H}}>4 \pi \ell^{2}$, where $M\left(A_{\mathrm{H}}\right) \sim A_{\mathrm{H}}^{3 / 2}$, we have $\Delta>0$ reflecting thermal stability. Since for this case $\Delta\left(A_{\mathrm{H}}\right) \sim A_{\mathrm{H}}^{-1}$, the canonical entropy for a large area stable AdS Schwarzschild black hole is given by $S_{\text {can }}=$ $S_{\text {micro }}\left(A_{\mathrm{H}}\right)+\frac{1}{2} \ln A_{\mathrm{H}}+\cdots=A_{\mathrm{H}} /\left(4 \ell_{P}^{2}\right)-\ln \left[A_{\mathrm{H}} /\left(4 \ell_{P}^{2}\right)\right]+\cdots$. This result, in fact, holds for any smooth mass function $M\left(A_{\mathrm{H}}\right)$. Now, in between the small and large area regions, the point $\Delta=0$ (heat capacity $C$ diverging) occurs at a critical value of the horizon area $A_{c}$ where the mass function equals the micro-canonical entropy in appropriate units, $M\left(A_{c}\right)=S_{\text {micro }}\left(A_{c}\right)$. This is the point of Hawking-Page transition. Thus a characterization of this phase transition without any reference to the classical metric has been obtained.

We close our survey with one last remark. The presence of the logarithmic correction in the asymptotic horizon entropy may have consequences in a variety of phenomenon in the theory of gravity. In particular, these may show up in cosmology. For example, its implications for entropic inflation have been explored recently in [55]. 


\section{Acknowledgements}

The author gratefully acknowledges collaborations with T.R. Govindarajan, P. Majumdar, S. Kalyana Rama and V. Suneeta which have lead to many of the results surveyed here. Thanks are also due to Ghanashyam Date for his useful comments. The support of the Department of Science and Technology, Government of India, through a J.C. Bose National Fellowship is gratefully acknowledged.

\section{References}

[1] Bekenstein J., Black holes and entropy, Phys. Rev. D 7 (1973), 2333-2346.

Bekenstein J., Generalized second law of thermodynamics in black hole physics, Phys. Rev. D 9 (1974), 3292-3300.

Bardeen J.M., Carter B., Hawking S.W., The four laws of black hole mechanics, Comm. Math. Phys. 31 (1973), 161-170.

Hawking S.W., Particle creation by black holes, Comm. Math. Phys. 43 (1975), 199-220.

Page D., Particle emission rates from a black hole: massless particles from an uncharged, non-rotating hole, Phys. Rev. D 13 (1976), 198-206.

Unruh W.G., Notes on black-hole evaporation, Phys. Rev. D 14 (1976), 870-892.

[2] Ashtekar A., Beetle C., Dreyer O., Fairhurst S., Krishnan B., Lewandowski J., Wiśniewski J., Generic isolated horizons and their applications, Phys. Rev. Lett. 85 (2000), 3564-3567, gr-qc/0006006.

Ashtekar A., Beetle C., Fairhurst S., Mechanics of isolated horizons, Classical Quantum Gravity 17 (2000), 253-298, gr-qc/9907068.

Ashtekar A., Fairhurst S., Krishnan B., Isolated horizons: Hamiltonian evolution and the first law, Phys. Rev. D 62 (2000), 104025, 29 pages, gr-qc/0005083.

[3] Kaul R.K., Majumdar P., Schwarzschild horizon dynamics and SU(2) Chern-Simons theory, Phys. Rev. D 83 (2011), 024038, 10 pages, arXiv:1004.5487.

[4] Birmingham D., Blau M., Rakowski M., Thompson G., Topological field theory, Phys. Rep. 209 (1991), 129-340.

[5] Kaul R.K., Govindarajan T.R., Ramadevi P., Schwarz type topological quantum field theories, in Encyclopedia of Mathematical Physics, Elsevier, Amsterdam, 2006, 494-503, hep-th/0504100.

[6] Rovelli C., Quantum Gravity, Cambridge University Monographs on Mathematical Physics, Cambridge University Press, Cambridge, 2004.

Ashtekar A., Lewandowski J., Background independent quantum gravity: a status report, Classical Quantum Gravity 21 (2004), R53-R152, gr-qc/0404018.

Theimann T., Modern canonical quantum general relativity, Cambridge Monographs on Mathematical Physics, Cambridge University Press, Cambridge, 2007.

Sahlmann H., Loop quantum gravity - a short review, arXiv:1001.4188.

[7] Basu R., Kaul R.K., Majumdar P., Entropy of isolated horizons revisited, Phys. Rev. D 82 (2010), 024007, 5 pages, arXiv:0907.0846.

[8] Engle J., Noui K., Perez A., Black hole entropy and $S U(2)$ Chern-Simons theory, Phys. Rev. Lett. 105 (2010), 031302, 4 pages, arXiv:0905.3168.

Engle J., Noui K., Perez A., Pranzetti D., Black hole entropy from an $S U(2)$-invariant formulation of Type I isolated horizons, Phys. Rev. D 82 (2010), 044050, 23 pages, arXiv:1006.0634.

[9] Ashtekar A., Baez J., Corichi A., Krasnov K., Quantum geometry and black hole entropy, Phys. Rev. Lett. 80 (1998), 904-907, gr-qc/9710007;

Ashtekar A., Corichi A., Krasnov K., Isolated horizons: the classical phase space, Adv. Theor. Math. Phys. 3 (2000), 419-478, gr-qc/9905089;

Ashtekar A., Baez J., Krasnov K., Quantum geometry of isolated horizons and black hole entropy, Adv. Theor. Math. Phys. 4 (2000), 1-94, gr-qc/0005126.

[10] Smolin L., Linking topological quantum field theory and nonperturbative quantum gravity, J. Math. Phys. 36 (1995), 6417-6455, gr-qc/9505028.

[11] Krasnov K.V., On quantum statistical mechanics of Schwarzschild black hole, Gen. Relativity Gravitation 30 (1998), 53-68, gr-qc/9605047.

[12] Rovelli C., Black hole entropy from loop quantum gravity, Phys. Rev. Lett. 77 (1996), 3288-3291, gr-qc/9603063. 
[13] Kaul R.K., Majumdar P., Quantum black hole entropy, Phys. Lett. B 439 (1998), 267-270, gr-qc/9801080.

[14] Kaul R.K., Majumdar P., Logarithmic correction to the Bekenstein-Hawking entropy, Phys. Rev. Lett. 84 (2000), 5255-5257, gr-qc/0002040.

[15] Das S., Kaul R.K., Majumdar P., A new holographic entropy bound from quantum geometry, Phys. Rev. D 63 (2001), 044019, 4 pages, hep-th/0006211.

[16] Kaul R.K., Kalyana Rama S., Black hole entropy from spin one punctures, Phys. Rev. D 68 (2003), 024001, 4 pages, gr-qc/0301128.

[17] Fursaev D.V., Temperature and entropy of a quantum black hole and conformal anomaly, Phys. Rev. D 51 (1995), R5352-R5355, hep-th/9412161.

Mann R.B., Solodukhin S.N., Universality of quantum entropy for extreme black holes, Nuclear Phys. B 523 (1998), 293-307, hep-th/9709064.

[18] Witten E., Quantum field theory and the Jones polynomial, Comm. Math. Phys. 121 (1989), 351-399.

[19] Witten E., On holomorphic factorization of WZW and coset models, Comm. Math. Phys. 144 (1992), 189212.

Blau M., Thompson G., Derivation of the Verlinde formula from Chern-Simons theory and the G/G model, Nuclear Phys. B 408 (1993), 345-390, hep-th/9305010.

[20] Kaul R.K., Chern-Simons theory, colored-oriented braids and link invariants, Comm. Math. Phys. 162 (1994), 289-320, hep-th/9305032.

Kaul R.K., Chern-Simons theory, knot invariants, vertex models and three manifold invariants, in Frontiers of Field Theory, Quantum Gravity and Strings, Horizons in World Physics, Vol. 227, Nova Science Publishers, New York, 1999, 45-63, hep-th/9804122.

Kaul R.K., Ramadevi P., Three-manifold invariants from Chern-Simons field theory with arbitrary semisimple gauge groups, Comm. Math. Phys. 217 (2001), 295-314, hep-th/0005096.

[21] Di Francesco P., Mathieu P., Senechal D., Conformal field theory, Graduate Texts in Contemporary Physics, Springer, Berlin, 1997.

[22] Livine E.R., Terno D.R., Quantum black holes: entropy and entanglement on the horizon, Nuclear Phys. B 741 (2006), 131-161, gr-qc/0508085.

[23] Domagala M., Lewandowski J., Black-hole entropy from quantum geometry, Classical Quantum Gravity 21 (2004), 5233-5243, gr-qc/0407051.

Meissner K.A., Black-hole entropy in loop quantum gravity, Classical Quantum Gravity 21 (2004), 52455251, gr-qc/0407052.

Khriplovich I.B., Quantized black holes, correspondence principle, and holographic bound, gr-qc/0409031.

[24] Ghosh A., Mitra P., An improved lower bound on black hole entropy in the quantum geometry approach, Phys. Lett. B 616 (2005), 114-117, gr-qc/0411035.

[25] Aref'eva I.Ya., Non-Abelian Stokes theorem, Theoret. and Math. Phys. 43 (1980), 353-356.

[26] Sahlmann H., Thiemann T., Chern-Simons theory, Stokes' theorem, and the Duflo map, J. Geom. Phys. 61 (2011), 1104-1121, arXiv:1101.1690.

Sahlmann H., Thiemann T., Chern-Simons expectation values and quantum horizons from LQG and the Duflo map, arXiv:1109.5793.

[27] Duflo M., Opérateurs differéntiels bi-invariants sur un groupe de Lie, Ann. Sci. École Norm. Sup. (4) 10 (1977), 265-288.

[28] Carlip S., Logarithmic corrections to black hole entropy from the Cardy formula, Classical Quantum Gravity 17 (2000), 4175-4186, gr-qc/0005017.

[29] Cardy J.L., Operator content of two-dimensional conformally invariant theories, Nuclear Phys. B 270 (1986), 186-204,

Blöte H.W.J., Cardy J.L., Nightingale M.P., Conformal invariance, the central charge, and universal finitesize amplitudes at criticality, Phys. Rev. Lett. 56 (1986), 742-745.

[30] Brown J.D., Henneaux M., Central charges in the canonical realization of asymptotic symmetries: an example from three-dimensional gravity, Comm. Math. Phys. 104 (1986), 207-226.

Larsen F., A string model of black hole microstates, Phys. Rev. D 56 (1997), 1005-1008, hep-th/9702153. Maldacena J.M., Strominger A., Universal low-energy dynamics for rotating black holes, Phys. Rev. D 56 (1997), 4975-4983, hep-th/9702015.

[31] Strominger A., Vafa C., Microscopic origin of the Bekenstein-Hawking entropy, Phys. Lett. B 379 (1996), 99-104, hep-th/9601029;

Horowitz G.T,, Lowe D.A., Maldacena J.M., Statistical entropy of non extremal four-dimensional black holes and $U$ duality, Phys. Rev. Lett. 77 (1996), 430-433, hep-th/9603195. 
[32] Birmingham D., Sen S., An exact black hole entropy bound, Phys. Rev. D 63 (2001), 047501, 3 pages, hep-th/0008051.

Birmingham D., Sachs I., Sen S., Exact results for the BTZ black hole, Internat. J. Modern Phys. D 10 (2001), 833-857, hep-th/0102155.

[33] Govindarajan T.R,, Kaul R.K., Suneeta V., Logarithmic correction to the Bekenstein-Hawking entropy of the BTZ black hole, Classical Quantum Gravity 18 (2001), 2877-2885, gr-qc/0104010.

[34] Achúcarro A., Towensend P.K., A Chern-Simons action for three-dimensional anti-de Sitter supergravity theories, Phys. Lett. B 180 (1986), 89-92.

Witten E., (2+1)-dimensional gravity as an exactly soluble system, Nuclear Phys. B 311 (1988), 46-78.

Carlip S., Entropy from conformal field theory at Killing horizons, Classical Quantum Gravity 16 (1999), 3327-3348, gr-qc/9906126.

[35] Carlip S., Teitelboim C., Aspects of black hole quantum mechanics and thermodynamics in $2+1$ dimensions, Phys. Rev. D 51 (1995), 622-631, gr-qc/9405070.

[36] Labastida J.M.F., Ramallo A.V., Operator formalism for Chern-Simons theories, Phys. Lett. B 227 (1989), 92-102.

Isidro J.M., Labastida J.M.F., Ramallo A.V., Polynomials for torus links from Chern-Simons gauge theories, Nuclear Phys. B 398 (1993), 187-236, hep-th/9210124.

Hayashi N., Quantum Hilbert space of $G_{C}$ Chern-Simons-Witten theory and gravity, Prog. Theor. Phys. Suppl. (1993), no. 114, 125-147.

[37] Carlip S., The statistical mechanics of the three-dimensional Euclidean black hole, Phys. Rev. D 55 (1997), 878-882, gr-qc/9606043.

Suneeta V., Kaul R.K., Govindarajan T.R., BTZ black hole entropy from Ponzano-Regge gravity, Modern Phys. Lett. A 14 (1999), 349-358, gr-qc/9811071.

[38] Govindarajan T.R., Kaul R.K., Suneeta V., Quantum gravity on $d S_{3}$, Classical Quantum Gravity 19 (2002), 4195-4205, hep-th/0203219.

[39] 't Hooft G., The black hole interpretation of string theory, Nuclear Phys. B 335 (1990), 138-154.

[40] Susskind L., Some speculations about black hole entropy in string theory, hep-th/9309145.

[41] Halyo E., Rajaraman A., Susskind L., Braneless black holes, Phys. Lett. B 392 (1997), 319-322, hepth/9605112.

Halyo E., Kol B., Rajaraman A., Susskind L., Counting Schwarzschild and charged black holes, Phys. Lett. B 401 (1997), 15-20, hep-th/9609075.

Horowitz G.T., Polchinski J., A correspondence principle for black holes and strings, Phys. Rev. D 55 (1997), 6189-6197, hep-th/9612146.

Damour T., Veneziano G., Selfgravitating fundamental strings and black holes, Nuclear Phys. B 568 (2000), 93-119, hep-th/9907030.

Halyo E., Universal counting of black hole entropy by strings on the stretched horizon, J. High Energy Phys. 2001 (2001), no. 12, 005, 15 pages, hep-th/0108167.

[42] Kaul R.K., Black hole entropy from a highly excited elementary string, Phys. Rev. D 68 (2003), 024026 , 4 pages, hep-th/0302170.

[43] Kalyana Rama S., Asymptotic density of open p-brane states with zero-modes included, Phys. Lett. B 566 (2003), 152-156, hep-th/0304152.

[44] Gour G., Algebraic approach to quantum black holes: logarithmic corrections to black hole entropy, Phys. Rev. D 66 (2002), 104022, 8 pages, gr-qc/0210024.

Gupta K.S., Sen S., Further evidence for the conformal structure of a Schwarzschild black hole in an algebraic approach, Phys. Lett. B 526 (2002), 121-126, hep-th/0112041.

Bianchi E., Black hole entropy, loop gravity, and polymer physics, Classical Quantum Gravity 28 (2011), 114006, 12 pages, arXiv:1011.5628.

[45] Davidson A., Holographic shell model: stack data structure inside black holes, arXiv:1108.2650.

[46] Agulló I., Barbero G. J.F., Borja E.F., Diaz-Polo J., Villaseñor E.J.S., Combinatorics of the $S U(2)$ black hole entropy in loop quantum gravity, Phys. Rev. D 80 (2009), 084006, 3 pages, arXiv:0906.4529.

Agulló I., Barbero G. J.F., Borja E.F., Diaz-Polo J., Villaseñor E.J.S., Detailed black hole state counting in loop quantum gravity, Phys. Rev. D 82 (2010), 084029, 31 pages, arXiv:1101.3660;

Engle J., Noui K., Perez A., Pranzetti D., The $S U(2)$ black hole entropy revisited, J. High Energy Phys. 2011 (2011), no. 05, 016, 30 pages, arXiv:1103.2723.

[47] Freidel L., Livine E.R., The fine structure of $S U(2)$ intertwiners from $U(N)$ representations, J. Math. Phys. 51 (2010), 082502, 19 pages, arXiv:0911.3553. 
[48] Sahlmann H., Black hole horizons from within loop quantum gravity, Phys. Rev. D 84 (2011), 044049 , 12 pages, arXiv:1104.4691.

[49] Krasnov K., Rovelli C., Black holes in full quantum gravity, Classical Quantum Gravity 26 (2009), 245009, 8 pages, arXiv:0905.4916.

[50] Agulló I., Barbero G. J.F., Díaz-Polo J., Borja E.F., Villaseñor E.J.S., Black hole state counting in LQG: a number theoretical approach, Phys. Rev. Lett. 100 (2008), 211301, 4 pages, arXiv:0802.4077.

Barbero G. J.F., Villaseñor E.J.S., Generating functions for black hole entropy in loop quantum gravity, Phys. Rev. D $\mathbf{7 7}$ (2008), 121502, 5 pages, arXiv:0804.4784.

Barbero G.J.F., Villaseñor E.J.S., On the computation of black hole entropy in loop quantum gravity, Classical Quantum Gravity 26 (2009), 035017, 22 pages, arXiv:0810.1599.

[51] Caravelli F., Modesto L., Holographic actions from black hole entropy, Phys. Lett. B 702 (2011), 307-311, arXiv:1001.4364.

[52] Das S., Majumdar P., Bhaduri R.K., General logarithmic corrections to black hole entropy, Classical Quantum Gravity 19 (2002), 2355-2367, hep-th/0111001.

[53] Gour G., Medved A.J.M., Thermal fluctuations and black hole entropy, Classical Quantum Gravity 20 (2003), 3307-3326, gr-qc/0305018.

[54] Chatterjee A., Majumdar P., Universal canonical black hole entropy, Phys. Rev. Lett. 92 (2004), 141301, 4 pages, gr-qc/0309026.

Chatterjee A., Majumdar P., Universal criterion for black hole stability, Phys. Rev. D 72 (2005), 044005, 3 pages, gr-qc/0504064.

Majumdar P., Generalized Hawking-Page phase transition, Classical Quantum Gravity 24 (2007), 17471753, gr-qc/0701014.

[55] Easson D.A., Frampton P.H., Smoot G.F., Entropic inflation, arXiv:1003.1528. 